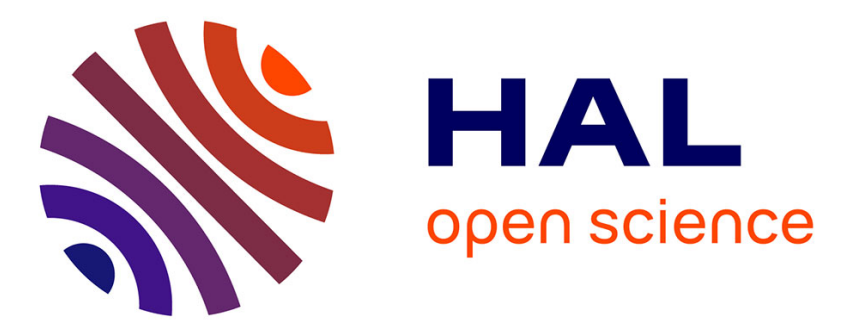

\title{
Recherches archéologiques en Gaule en 1951 (suite)
}

Raymond Lantier

\section{To cite this version:}

Raymond Lantier. Recherches archéologiques en Gaule en 1951 (suite). Gallia - Fouilles et monuments archéologiques en France métropolitaine, 1953, 11 (2), pp.327-362. 10.3406/galia.1953.1354 . hal01921083

\section{HAL Id: hal-01921083 \\ https://hal.science/hal-01921083}

Submitted on 4 Feb 2020

HAL is a multi-disciplinary open access archive for the deposit and dissemination of scientific research documents, whether they are published or not. The documents may come from teaching and research institutions in France or abroad, or from public or private research centers.
L'archive ouverte pluridisciplinaire HAL, est destinée au dépôt et à la diffusion de documents scientifiques de niveau recherche, publiés ou non, émanant des établissements d'enseignement et de recherche français ou étrangers, des laboratoires publics ou privés.

\section{(이)(\$)}

Distributed under a Creative Commons Attribution - NonCommercial - NoDerivatives| 4.0 


\title{
CHRONIQUE DES PUBLICATIONS
}

\author{
RECHERCHES ARGHÉOLOGIQUES EN GAULE EN 1951 (SUITE) *
}

(PÉRIODE HISTORIQUE)

\section{Époque Galio-Romaine}

1. La Conquête. Truvaux militaires. Le bimillénaire d'Alésia a suscité de nouvelles recherches portant sur les campagnes césariennes. J. Toutain 1 reprend l'examen de la bataille du Mont Rhéa, s'appuyant sur le texte des Commentaires et les découvertes archéologiques pour réfuter, une fois de plus, l'affirmation selon laquelle Alésia ne serait pas l'oppilum du siège. La bataille de cavalerie, antérieure à la retraite dans Alésia, serait à situer 2 sur les rives de l'Armançon. Quant aux chiffres proposés par César pour l'armée de secours, ils seraient for"tement exagérés. Si l'on admet que le nom des Séquanes a pu ctre engendré par celui de la Seine et que leur territoire se serait étendu en Franche-Comté, encore au temps de la Guerre des Gaules, sur la rive droite de la saòne, peut-être jusqu'aux sources de la scine, on disposerait

(*) V. Gallia, XI, 1, 1953, p. 174 sq.

(1) J. Toutain, $X X^{\mathrm{e}}$ congrès assoc. bourguignonne. soc. sav. Semur-en-Auxois, 1949 . p. 51-5\%.

(2) Gl. Leleu, ibid., p. 27-36; $\Lambda$ bbé JoviGNOT, Bull. soc. sc. Se'mur, 1951, n० 3. d'un nouvel argument en faveur de l'identification Mlise = Alćsia, puisque Gésar localise le combat de cavalerie à la fois au voisinage de la frontière des Séquanes et près d'Alésia. Il y a hien peu de renseignements à retircr d'une note sur les travaux de César devant $A$ lise ${ }^{3}$. A propos de la bataille de Gergovie, P.-F. Fournier 4 s'inscrit en faux contre le parti pris par César de minimiser son intention d'offensive contre la forteresse, qui n'aur'dit été qu'un simple coup de boutoir contre les premières défenses gauloises pour en éprouver la résistance. Au vrai, César voulait prendre la place, sans quoi son armée n'aurait pas ćté dotée d'un matćriel d'assaut aussi puissarit.

On ne s'étonnera pas que ce bimillénaire ait fourni l'occasion, vaine d'ailleurs, de rompre de nouvelles lances pour ou contre les fastidicux problèmes, déjà résolus, de la localisation de tel ou tel site célèbre de la guerre des Gaules. Si cela était encore nécessaire, des arguments toponymiques 5 sont jetés dans la bataille

(3) R.-P. Noche, $X X^{e}$ congrès assoc. bourg. soc. sav. Semur, 1949, p. 27-36.

(4) P.-F. Fourvier, Bull. soc. hist. et scientif. Auvergne, LXXI, 1951, p. 67-68.

(5) P. LEBEL, $X X^{\mathrm{e}}$ congrès assoc, bourg. soc. sav. Semur, 1949, p. 29-36. 
qui met aux prises Bourguignons et Francs-Comtois. Toponymie et phonétique prouvent qu'Alaise ne peut etre Alésia : Alesia serait la forme lingonne, Alisia la forme mandubienne. Le nom d'Alésia aurait été répandu, dans le monde antique, par les Commentaires, mais la forme locale Alisia se serait conservée jusqu'à nos jours. A l'histoire des fouilles d'Alise-Sainte-Reine, il faut ajouter de nouvelles contributions: la découverte de lettres inédites de stoffel, qui dirigea les recherches de 1861 à 1865, et d'un plan partiel des travaux à la date

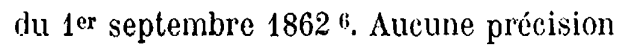
n'est, par contre, apportéc par un article sur les circonstances de la découverte, dans les travaux de circonvallation, du fameux vase d'argent 7. G. Grémaud a réuni d'anciennes gravures représentant le site d'Alésia 8.

Malgrí les découvertes récentes faites a l'Impernal de Iuzech, dans les secteurs Ouest et Nord de l'oppidum, du côté de la Combe des Chônes 9 , d'un temple ì double enceinte du genre fanum, contemporain du I $^{\text {er }}$ siècle de notre ìre, d'un bâtiment rectangulaire, de soubassements carrés et de céramiques hallstatticnnes, campaniennes et gallo-romaiises du début de l'Empire, l'identification du site aver: Uxellodunum n'emporte pas un aquiescement unanime. Pour certains l'emplacement de la ville gauloise est à rechercher a Capdenac, où se trouve la "vraic fonlaine" de tous les points conformes au récit d'Hirtius et dont le voisinage aver: l'Auvergne permit à Jucterius d'échapper au combat devant la forteresse ${ }^{10}$. Il en̂t clé étonnant que, dans cette vaine que-

(6) Abbes Jovig.vor, ibid., p. 80.

(7) A. Collet, ibid., p. 59-65.

(8) G. Gremaun, ibid., p. 37-40.

(9) M. Labroussli, Bull. soc. d'ét. Lot, 19:i)l. p. 54 .

(10) I. Cons, ibid., p. 23-26. relle, le nom de Murcens u'ait pas été, lui aussi, proposé 11.

I ad guerre des Helvètes fait entrer Lyon dans l'histoire 12 : César établit son premier camp à Fourvière, sur le plateau de. la Sarra, bien desservi par le chemin qui le traversait, avec la possibilifé d'utiliser le port de Choulans pour l'acheminement du ravitaillement venu de Provence. Après le départ de César, le camp de Fourvière n'aurait pas été abandonné, et ses vétérans furent pourvus de terres. Avec les réfugiés de Vienne, les légionnaires císariens furent les premiers occupants du lyon romain. La démonstration est ingénieuse, mais ne sort pas du domaine de l'hypothèse: interprótation des textes historiques et de l'inscription de la rotonde de l'Antiquaille à Fourvì̀re fixant les limites territoriales d'un collège des Iares, établi dans une domus Juliama, qui ne serait autre que la demeure de César lors de son séjour au camp et pendant lequel le proconsul aurait cu l'intuition des possibilités offertes par le site de Iyon. C'est ainsi que s'expliquerait son ('hoix de Lucius Munatius l'lancus pour le gouvernement, en 43 , de la Gaule Chevelue. Plancus serait l'homme yui aurait assurć les projets de César sur Iyon. L'assassinat du dictateur ruine ses projets; Plancus mène campagne contre les Rhìtes de la Suisse Orientale, victorieux il regagne la Gaule et ce serait au camp de Lugdumum qu'il fixe sa résidence. A cette occasion fut énise la monnaie de bronze à la légende Copia Folix Munatia, antérieure à la fondation de la colonie. Il faudrait donc dater cette piece du serond semestre de 44 ou du premier de $\mathbf{4 5}$. et admettre que Plancus, dès ce temps, arait établi une ville sur l'emplacement du camp cósarien, et c'est là, à Copia

(11) Le Parisien libéré, 25 déc. 1950.

(12) A. Auprs, César et Plamcus à Lyon, Lyon. 1951. 
Felix Munatia, qu'il aurait résidé jusqu'aux événements de 43 et ćchangé ses lettres avec Cicćron. I'histoire de la fondation de la colonie lyonnaise serait ansi intimement liće à celle des triumvirs, Antoine, Octave ct Lépide. Le 7 mars de l'annóc 43, plancus donnait une réalití all projet de César.

ra colonie se présente sous la forme d'un vaste triangle reclangle, dominant au Nord la Saône au-dessus du défiló de licrrc-Seize, de l'éperon de loyasse jusqu'à celui descendant de Fourvière sur Saiut-Paul (long. $11.000 \mathrm{~m})$, couronnant à l'Est le versant du confluent depuis l'échine de Saint-P'aul jusqu'i Saint-Just, passant au-dessus de Fourvière par le versant de l'Antiquaille $\left(17.050^{\mathrm{m}}\right)$. Ia fare Sud-Oucst longeait le rebord du plateau, utilisant le profond fossé du ravin de Choulans, ceux du Pré et do la Gorge du I.oup.

C'est encore une garnison militaire qui ost aux origines de Toulouse galloromaine 13. Vers 120 av. J.-C., après la gucre des Cimbres, le consul Cépion construil un castellum, dont les traces apparaissent peut-être sur l'emplacement de quatre petites rues en cul-de-sac formant une sorte de village dans un coin de Toulouse, avee deux seules issues, l'une sur la rue de la Fonderie, l'ancien chemin du gué, l'autre débouchant, en sens opposé, sur la Garonne.

2. Le's villes galio-romaines. - A Toulousccit, la cité paraît s'êtro développée par la concrétion el la progression d'éléments disséminés sur la rive gauche de la Garomne: le quartier d'Antipolis, en bordure de la première terrasse, principalement vers le confluent du Touch, et entre les quartiers de Saint-Michel et de

(13) M. BRoËNS, Mém. présentés par divers savants a l'Acad. des Inscr. et B.-L., XIV, ?c part., 1951, p. 287-314.
Saint-Étieme, site du forum et de la ville marchande. Ces deux centres, près des nouds routier's et du gué sur le fleuve, tendront à se grouper; celui de la rive droite, le plus développé, absorbe lia population de l'emporizm, l'autre is Antipolis(?), en aval sur l'autre berge, ne scra guère qu'un satellite. Avec le rir" s., la ville reçoit un nourel apport de population et il est à remarquer que les invalsions sont loin d'avoir étí une cause de ruine pour Toulouse, enfermée dans son rempart. La convergence systimatique de toutes les voies urbaines vers les išsues de la cité montre l'altraction cheore exercée par la banlieue anrès la construction des murailles, car langglomeririlion est loin de se limiter ì la ville close, elle comprend aussi les villas de la plaine et les vici alignés sur les terrasses do la valléc. Tout cela explique l'importance de l'enceinte $(2.900 \mathrm{~m}$. en demi-cercle), ouverte sur la Garonne, circonscrivant une ćtendue de 80 hectares, flanquant à l'Ouest lo quadrilatère du castellum, hérissé de 60 tours. Toulouse sera la capitale des rois goths. Ia conquate franque amènera sa déchéance.

Les origines de Narbonne 15 se rattachent aussi à l'cxistenre d'un oppidum : Nerênen, où l'on battait monnaise, situé au roisinage de l'emplacement où s'élèvera la colonie romaine, peut-être à Montlaulrès où ce numéraire ne manque pas. Un même fait se reproduira lors de la fondation d'Augustodunum qui, cent ans plus tard, remplacera Bibracte. Par la fondation de Narbonne, Rome a cherché à protéger la route de Taraconnaise qui ne pouvait rester sous la menace do groupes ayant des affinités avec les populations espagnoles, ou les roitelets celt1ques de loyauté douteuse. Nécessité de créer un centre de communications, un noud routier; or le site de Narbonne ré-

(15) A. Blanchet, C.R.A.I., 1951, p. 165. 
pond à ces conditions sur le passage de très anciens chemins reliant les pays des Tolosates aux rivages de la Méditerranée ¿ travers la vallée de l'Aude.

Il en est de même de Paris, dont la genèse et l'avenir dépendent d'un carrelour 16. The ligne de scuils presque imperceptibles relient, à travers l'Ile-deFrance, le Cambrésis à la Beauce et au Poitou, en un point où l'île de la Cité et Ia Montagne Sainte-Geneviève offrent les conditions les plus sûres pour la traversće du vaste marécage s'étendant sur l'emplacement des Grands Boulevards et. du Quarticr I atin. Trois routes maîtresses 17 y passent, unissant les Pyrćnées au Rhin, les Alpes à la Manche, les Vosges à l'Atlantique. Ces Iraits de structure géologique ont fixé les directions générales de ce grand passage 18 , et c'est encore à eux que la ville doit son supporl compact, le calcaire marin de paris, pliteau en forme de socle, où l'ćrosion fluviale a sculpté dans sa masse la Montagne Sainte-Geneviève et la hutle de Chaillot. Ils lui apportent aussi le gypse de la butte Montmartre ol les sibles de ses forêts lui fournissant ses matériaux de construction. De la plaine picarde à la plaine beauceronne l'itinćraire le plus facile pour franchir la Seine est le passage descendant par le col de la Chapelle et la montée de Sainte-Geneviève, la route de Saint-Martin et de Saint-Jacques. Aux origines de I,utèce, il y a les ponts, et si le site est submersible la protection des deux bras du fleuve lui permet le contrôle des grands chemins. Aux temps romains, le développement de

(16) A. Varaginac, Inst. internat. d'archeocivil. Extrt: L'age nouveau, 4 p., 1 carte du carrefour de Lutèce à l'époque gallo-romaine.

(17) M. Roblin, Histoire locale, III, 1951, $\mathrm{n}^{\circ}$ 5-8, p. 23.

(18) R. Dion, La Revue des Deux-Mondes, f'r janv. 1951, p. 4-30. la ville sur la rive gauche est en rapport avec le terminus de la roule de Rome. Aux temps troublés, la vie se concentrera de nouveau dans la Cité.

Le passé de Strasbourgr n'est pas moins ancien ${ }^{19}$. Sous la couche de tourbe ì tessons celtiques, des graviers recouvrant un lit de bois provenant des caillebotis de l'âge du Bronze (rue du Sanglier) correspondent à unc occupation du site par les Protoceltes; inondé el submergí par do. fortes crues du Rhin, il fut déserté environ 1100 avant l'ère, puis converti en marais pendant le Hallstattien. Ce ne fut gue pluss tard, à la faveur de l'assèchemont du climat, que les colles de Ia Tène s'installèrent à Strasbourg. Tes découvertes récentes, - dégagement ì l'angle de la rue des Juifs et de la l'ue du Dôme, d'une partie du prítoire légionnaire, fouilles à l'intérieur de l'angle Fst du camp, sous la ruelle actuelle de saintMédard, photographies et ohservations aériennes ayant révélé la présence d'un camp contemporain de la conquête des Champs Décumates au sud de la Meinau, - ont apporté des documonts nouveaux i l'histoire de Strasbourg, dont le camp et l'agglomération furent détruits en 70 ct en 97 ap. J.-C. Une période de paix, de 120 à l'êpoque des Sévères, est marquée par l'abandon du Camp envahi alors par la population. Au cours des mutineries consécutives à la mort d'AlexandreSévère, les Alamans ravagent la cité. Maximien rétablit le camp, et le rèone de Constantin est marqué par des remaniements et transformations. Nouvelle destruction de la ville par les Alamans et reconstruction sous Valentinien. Au $\mathrm{v}^{\mathrm{e}}$ siècle, l'Alsace est envahie et aucun vestige mérovingien n'apparait dans l'enreinte. Ta ville sera réoceupée lors de la renaissunce carolingienne. Aux temps ro-

(19) J.-J. HaTT, Rev, archéol. Est, II, 1951, p. 121 sq.; Gallia, VII, 2, 1949, p. 161-189. 
mains, l'agglomération comprend deux villes distinctes : l'ancienne cilé gauloise, incluse dans lc camp militairc, Argcncorate, la ville forte ct les canabae avec le virus de Koenigshofen, la ville routière, le long de la grandroute, strataeburgus, oì s'installeront les Germains dont on connait l'horreur pour les sites entourés de remparts.

line esquisse de l'évolution urbaine d'Autun, depuis sa fondation 20 , pose plus de problèmes qu'elle n'en résout. Ià chcore, on se trouve en prósence d'une ville de carrefour sur des voies naturelles entre les bassins de la Loire, de la seine et du Rhône, sur un plateau dominant la vallée de l'Arroux. La fondation augustéenne de 15 av. J.-G. s'applique à un terrain neuf : un plan en damier circonscrit par une enceinte, mais l'irrégularité du support impose au quadrillage une certaine souplesse. La ville haute s'étage en gradins réunis par des escaliers, et sur ta plus haute plate-forme le capitole, le temple d'Apollon, puis les écoles au $\mathrm{II}^{\circ}$ siècle. Au champ Saint-Iadre, ce seront le forum et les édifices publics. On est en prósence d'une ville type móditerrancen, dont les grandes demeures, ive? leurs jardins clos de murs légers et sans fondations, occupent des îlots enlicrs, les industries métallurgiques dessinant une sorte de ceinture intérieure le long des remparts. Après les ravages du siège de 269, Autun se relève, mais reste isolé au milieu d'une campagne ravagée. Ia construction dans la ville haute d'une nouvelle muraille, à la fin du IV siècle, amène la destruction des monuments publics. Rien ne permet d'affirmer que la ville basse ait été alors abandonnée. Le rastrum ne fut qu'un lieu de défense. Si la population a pu diminuer, elle ne pa-

(20) J. Berthollet, Mém. soc. éduenne, L, 1950-1951, p. 165-192. rait pas avoir changé ses habitudes et n'a pas cherché à s'agglomérer dans un étroit pórimètrc.

Les origines de Dijon 21 sont, clles encore, antérieures à l'époque romaine. La bourgade était placćc au croisement de plusieurs chemins très ancicns, l'un longeant le revers du plateau bourguignon, les autres menant de la saone à l'intériecu du plateau notamment ver's la Sejne. Importante au Haut Empire, la ville se resserre dans le castrum dressé sur la colline, à quelque distance, lors des invasions.

I.cs travaux de reconstruction et d'aménagement sont, comme chaque annóc, la source de nouvelles découvertes. La continuation des recherches à Marseille ${ }^{22}$, dans les quartiers du forum et du théâtre, sur le littoral du Jacydon, ont prouvé qu'à l'époque grecque la ligne du rivage s'infléchissait en suivant les courbes de niveaux de la Butle des Moulins, pour donner naissance à une anse assez profonde au pied de la place de Ienche. Les nivellements de 49 avant l'ère ont alors transformé le profil du rivage par des apports de sables et de graviers marins, et de déblais provenant de l'arasement de la Butte Saint-Taurent. Ces terrassements et ces comblements ont sensiblement boulcversé la lopographie urbaine par le comblement d'une anse à l'angle des rues des Marligales et de Sclvian de la Figuière, pour l'assise du dallage du forum et d'une calcnque, au pied de la Butte Saint-Laurent, pour la consIruction du théâtre. Do nouvelles superpositions ont été retrouvces, rue Négrel, aux abords du four gree dójà connu, audessus duquel s’éleva une construction

(21) P. Gras, Rev. ét. lat., $\mathrm{XXIX,1951,}$ p. 66-67.

(22) F. BevoIt, Gallia, VIII, 1950, p. 116117. 
d'époque hellénistique. Dans le bas de la rue Négrel, à trente mètres de la Grande Rue, sous une mosaïque s'étend une assise de la muraille, épaisse de $1^{\mathrm{m}}, 70$, à parement de gros blocs équarris à l'extérieur, plus petits à l'intérieur, alignés Nord-Sud, suivant le tracé de la rue (IV siècle). Sur le plateau de la Butte Saint-Iaurent, on a reconnu la présence de sols en béton romain de tuileaux et au Midi, d'un dépotoir du rer siècle au pied de la butte. Place de Ienche ${ }^{23}$, des murs isodomes appartenant à un édifice antique ont été découverts dans les caves du monastère des religieuses de Saint-Sauveur, itroites et longues casemates relices par une sorte de couloir s'ouvrant au fond sur le port.

A Aix-en-Provence (Bouches-du-Rhônes) ${ }^{24}$, des sondages dans la partie méridionale du Jardin de Grassi ont fait connaître un béton de mortier supportant une dalle et un tronçon de colonne; au Glos Borel, des tessons d'Arezzo et de Ta Graufesenque et les vestiges d'un mur de basse époque.

On annonce à Arles. (Bouches-du-Rhône) le dégagement de l'hyposcenium du théâtre et la découverte, à l'extrémité septentrionale, de fosses en partie taillées dans le roc, dont la paroi sud, inclinée en pente douce, supportait un escalier de bois desservant les sous-sols du proscenium, constitué par un ban du rocher recouvert d'un plancher 25 . Ie dégagement de la galerie Nord des cryptoportiques a mis au jour l'un des deux couloirs des horrea, avec sa porte à linteau, ouverte sur le decumanus et encadrant des constructions annexes, plaquécs sur cette façade sous le règne de Constantin. Ces couloirs paraissent avoir tité les seuls accès de ces galeries, dont l'aile Nord était de plain pied avec la cité.

(23) Le Méridional, 22 déc. 1951.

(24) F. Bromort, Gallia, VIII, 1950, p. 119.

(25) DU MENE, ibid., p. 120.
Dans le dépotoir de marbres, provenant sans doute du temple de la Gens Augusta, on a recueilli une tête d'Octavien avec la barbe, deux mains et une copie de 26 av. J.-C. du clipcus virtutis d'Auguste ${ }^{26}$. Il n'y a pas eu de trouvailles nouvelles a Trinquetaille dans les sondages du terrain limitrophe au Rhône, au Nord et au Sud du pont. Te quartier résidentiel était concentré dans une partie du faubourg do la verrerie et au quartier du Gallègue. Au château des Baux, uà groasse tour était assise sur une construction romaine et le grand monument a are de décharge, en bordure de la rue des Cuiratiers.. avait été utilisé au Moyen âge. Dans l'enclos Clair, une villa rustique a ité restaurée au Bas Empire, et au pied du talus de la Gare Maritime, un premier sondage laisse entrevoir les traces d'une ocupation próromaise de Trinquetaille 27.

Les fouilles de 1950 à Sint-Remy-deProvence (Bouches-du-Rhome) as onl porlé au Sud-Ouest de l'agglomération : dégagement d'une grande cour i portique appartenant à un ensemble ornemental. large mur se dóveloppant cn une enceinte rectangulaire ouverte à l'Est et substruclions de deux édifices culturols en bel appareil de moellons smillés, avec chaînage de grand appareil aux angles et couronné par une corniche à modillons et décor floral, sommée en doucine de têtes de lions. Jess deux édifices juxlaposés, la façade à l'Est, appartiennent à deux périodes de construction, l'assise inféricure est du ier siècle, au II ${ }^{\mathrm{e}}$ siècle la partie infórieure du podium fut surélcrée et encadrée d'une large moulure au talon. Si les dimensions varient, le décor est le même, l'un et l'autre prostyles, paraissant. tétrastyles. I'ordre est corinthien. I'un a conservé les fragmente de la stalue de

(26) Ibid., p. 120.

(27) Ibill., p. 120-121.

(28) II. ROLLLAND, ibid., p. 131-132. 
la cella, jeune togatus cn marbre blane, debout, une capsa à ses pieds et une bulle d'or au cou. La partic Sud du péribole repose sur un édifice de Glanum I, une salle de réunion avec gradins et dans la muraille ont été réutilisés des autels aux Junones et un autre portant une inscription celtique. A l'Est, un bassin carré précède une exèdre, ornce d'une frise de boucliers, cuirasse ct denx captifs attachés à leur bouclier ovale.

a Fréjus (Var), dans le quartier du Moulin-à-Vent 29 au Nord-Ouest de la ville, on mil au jour des cilernes, des piseines, des canalisations, des pavements bétonnés ct, parallèle au decumanus, orientée Nord-Est - Sud-Est, une salle dont le sol est revètu d'un dallage en losanges de marbre blanc encadrant un motif carré de schiste gris, avec entourage de marbre blanc scgmenté. Au Nord de l'hôpital, le tracé de la voie décumane a été reconnu, ainsi que l'égout des cardo secondaires au Nord de Saint-Françoisde-Paule.

Divers monuments antiques ont été repérés à Valence (Drorne) $)^{30}$ : un théâtre près des bâtiments de la Prćfecture, dont l'axe est marqué par la côte SainteUrsule, adossé à la terrasse de la vilie haute: une habitation avec mosaiques, bassin et peut-être jardin au quartier Saint-Jacques; tuiles et fragments d'inscriptions près de Saint-Valcntin; ruines et statuettcs de bronze de Sucellus au quartier des Marais; substructions à Beauvallon et à Livron.

Les limites septentrionales et occidentales ont été atteintes dans la maison gallo-romaine de Ia Villasse à Vaison (Vaucluse), dont l'atrium et le bain avaient été précédemment dégagés : ensemble de salles desservies par un cou-

(29) F. BenoIt, ibid., p. 127.

(30) J. SAutel, ibid., p. 135-136. loir se terminant par les latrines. Pour n'être pas d'ordre spectaculaire, les découvertes faites dans les fondations de la cathédrale de Vaison offrent un grand intérêt pour l'histoire du site ct de la ville romaine. De ces recherches il apparait que cet édifice fut construit sur les ruines d'un grand monument romain dont on ne peut encore préciser la nalure, temple ou basilique d'une architeclure très soignće. Ine conséquence assez imprévue est que ce quartier chrétien, qui semble ćtabli à la périphéric, était plutôt lc centre de la (ité aux temps gallo-romains 31 . Sur la colline Saint-Jacques, au-dessus du vieil ermitage, à Cavaillon (Vaucluse) ${ }^{32}$, ce sont des restes próromains de remparts, des traces d'appartements, un fonds de cabane à tessons campaniens et drachmes de Marscille au taureau; place du Clos, près de l'arc de triomphe, une mosaique à décor géométrique noir et blanc, et à la maison $A$ pocco, des substructions.

Les fouilles d'Eauze (Ger's) $: 3$, sur le plateau de Cicutat, ont confirmé la présence des ruines de la ville romaine sur la terrasse de la Gélise, s'étendant sur un assez large périmètre entre la rivière. la roule de Condom et la voie ferrée, le centre de l'aggloméralion pouvant être recherché entre la gare et le château de Cieutat. Ies fouilles de Las Plassos ont montré que la ville avail été bâtie en damier, les rues se coupant à angle droit, délimitant des îlots carrés ou rectangulaires. Il s'agit donc d'une ville neuve et non de l'aménagement d'une bourgade préromaine, construite vers le milieu du ${ }^{\text {er }}$ siècle et détruite au $\mathrm{v}^{*}$.

Vayres (Gironde), lancienne Varate(31) Ibid., p. 144-145; C.R.A.I., 1951, p. 427432.

(32) DU MÊME, Gallia, VIII, 1950, p. 138.

(33) M. Labrousse, Bull. soc. archéol. Gers, LII, 1950, p. 201-213. 
do 34 , était un petil village de pêcheurs, de bateliers et de laboureurs, lorsque les Romains en firent une station routière au passage de la Dordogne, sur la route de Bordeaux à Périgueux, dont les rentres sont situés sur l'emplacement de l'église et du château et peut-ĉtre aussi du prieuré et du port de Saint-Pardoux. Entre ces deux points des villas sont dissćminées dans la campagne.

Ies fouilles du nouveau marché SaintPierre à Glermont-Ferrand (Puy-deDôme) 3 is ont fait comaître, à une profondeur de $5 \mathrm{~m}, 50$ sous le sol moderne, les vestiges d'un quartier occupé du $\mathrm{I}^{\mathrm{er}}$ au $\mathrm{I}^{\mathrm{e}}$ siccles. Les accumulations de cendres peuvent représenter des traces d'incendie nussi bien que l'accunulalion des déchıts de la vie quotidienne. Des substruptions ont été relevées à Poitiers (Vienne). rue du Grand-Cerf et au confluent de la Vienne et du Clain ${ }^{36}$, a ansi que l'empiervement de la voie romaine Genabum-Agedincum, à Orléans 37 .

Deux essais de reconstitution topographique sont proposés pour les villes gallo-romaines de Remes (Ille-rt-Vilaine) 38 et d'Avranches (Manche): :9.

En plusienrs poinls de Sens (Yonne). des travalux ont révélé le lracé de la voie de Sens à Troyes sous le pavement de la rue Thénard; un vaste édifice avec pièces ornées de niches place Drapès, près de l'emplacement présumé du forum, et à quatre-vingts modres au delà une salle

(34) A. Videau, Ilistoire de Vayres, Libourne, 1951.

(35) P.-F. Founvien, Bull, hist. et scientif. Auvergne, LXXI, 1950, p. 74-96.

(36) Bull. soc. antiq. Ouest, 1951, p. 591

(37) R. Lovis, Gallia, VIII, 1950, p. 168-169.

(38) A. Lovibard-Jourdan, Mem. soc. d'hist. et d'archéol. Bretagne, XXX, 1950; Bull. et mém. soc. archéol. Ille-et-Vilaine, I,XVIII, 1951, p. $37-38$.

(39) J. Mathine, Rerue de l'Atranchin, XXXVI-XXXVIII, 1950-1951, p. 614-622. avec hypocauste, dont les pilettes sont faites de tubuli remplis de mortier; débris de constructions Grande-Rue, rue Ahélard et rue Allix 40 .

Ia poursuite des fouilles dans les parcolles 397 et 398 du cadastre, à AliseSainte-Reine (Còte-d'()r) ${ }^{41}$, ont amené lo dégagement d'un quarticr de caractère assez particulier, résidentiel, formé do vastes et confortables demeures, avec (aves et liypocaustes, mais ne disposait pas de cours intérieures.

Fin 1951, le plan des thermes de la rue de Bcauvais à Amiens (Somine) 42 a pu être précisé : symétriquement à la salle à abside sur hypocauste, se développe à '()uest un frigidarium avec piscine dallée el entourée d'une banquette, au Nord un aqueduc et le caldarium. Ie mur, dérouvert place $\mathrm{I}_{\text {. de } \text { Wailly, appartiont à }}$ l'enceinte gallo-romaine, dont le tracé, partiellement repéré en ligne droite, an Nord et à l'Est de ce point, s'infléchit an sud pour épouser le contour septentrional de la rue Gresset. La face orientale, parallèle à la rue des Sergents, peut être datce par des monnaies de l'robus. Iienreinte était alors sensihlement moins importante, passant plus à l'()uest qu'on ne le supposait. Ia cathédrale et SaintRémy n’y sont point compris: mais elle englobait lous les monuments du centre de la ville antique, s'appuyant près de l'Hôtel de ville sur le théatre, dont les substructions ont été reconnues en deux points. Ia reprise des fouilles sur le sile de Baudimont a fait connaître une helle cave avee niches et soupirail de la fin du I $^{\text {er }}$ siècle de notre ère, remblaye sous les premiers Antonins, puis recouverte de constructions qui disparurent dans un incendie.

(40) R. Lours, Gallia, VIII, 1950, p. 178-180.

(41) L. LERAT, ibid., p. 155-158.

(42) J. Heurgon, L’Antiq. class., 1952, p. 139-140. 
Les travaux qui se poursuivenl à Bavai (Nord), dans les soü-sols du grand édifice en cours de dégagement ${ }^{43}$, confirment le caractère svstématique des transformations par lesquelles, après l'invasion qui le ruina entre 250 et 260 , le monument fut aménagé en place-forte, le sous-sol remblayé et l'ensemble entouró d'un rempart. Parmi les ohjets recueillis figure une applique de bronze: busto d'Atlis avec la pomme de pin et Cybèle, accostée de ses lions, de travail local.

3. Les monuments. - L'inscription de lat Porle d'Auguste à Nîmes (Gard) mentionne la construction par cet empereur de portes et de remparts. Il en est de mome du texte de la porto de Vienne (Isère) et on est alors en droit de conclure à une pacification encore lrop récente des territoires pour laisser sans défenses les colonies nouvelles ${ }^{44}$. Cette même porte d'Auguste, à Nîmes, est dotéc à ses deux extrémités de deux passages pour piétons couronnés chacun par une niche et ouverts entre deux pilastres corinthiens. Au centre, deux grandes arcades pour l'avaliers et véhicules sont surmontées d'un entablement supporté par deux taureaux dans l'axe de leur clé et, au milieu de l'ensemble, par le chapileau d'une colonnetle, dont la base repose sur une saillie de l'imposte des grandes arcades, qui soutient une pile quadrangulaire descendant jusqu'au sol ${ }^{45}$.

Ia restauration complète de l'arc d'Orange (Vaucluse) est en cours d'exécution 46 et elle prósentera l'avantage de pallier aux maladresses et aux malfaçons des réfections du sic̀cle dernier, nécessitant une reprise générale pour rejointoyer, enlever les "bouchons", rempla-

(43) Ibid., p. 140.

(44) J. Fonmigé, Rev. archéol., 1951, 2, p. 60-61.

(45) Ibid., p. 59

(46) Ch. P(ICARn), ibid., I, p. 220. cer les blocs rongés, restituer les moulures sur les faces méridionale et orientale. I.e sol et le rond-point devront être aussi aménagés. Sur l'architrave au sud courait une inscription en lettres de bronze répétant celle de la face septenlrionale.

Après avoir déterminé le tracé ct les extrémités du decumanus et du cardo de Vienne (Isère), on s'est efiorcé de retrouver l'emplacement probable de l'arc de la colonie romaine, placé à l'extrómité des voies, hors du rempart et vraisemblable. ment à leur intersection avec le pomarium. ${ }^{47}$. Le lieu le plus favorable pour une telle construction ne pouvait être que les extrémités du decumanus, l'une vers le pont au dolà duquel commencent les villas, l'autre dans l'étroite vallée de la Gère, et pour le cardo, la partie méridionale entourće de villas et menant au cirque, ou celle du Nord également visible du Rhône, dégagée de toute construction et tournćc vers Iyon. Ice problème semble maintenant résolu en favour de la localisation de l'arc sur le cardo, au Nord du rempart et au bord du fleuve, par la découverte ancienne d'éléments d'architectures ayant appartenu à un arc de triomphe, dressé au temps d'Auguste, renversé dans le Rhône et emporté par une crue: dauphins de bronze; corniche ayant pu faire partic des rampes d'un fronton de même profil qu'à Orange; deux pièds de chevaux en bronze; une épaule gauche drapée avec le bras et la main d'un conducteur de char ayant couronné un édifice; et dans les fondations du Palais épiscopal, celle de fragments de statues de captifs et de Victoires, de dieux marins, de monceaux d'armes, lance, épées, casque, bouclier, enseigne, et d'un panneau représentant des soldats.

Dans l'architecture des monuments de la Narbonnaise, à Vienne et à Nîmes. au

(47) J. Formicé, ibid., p. 62-64. 
temple de la Fontaine, des colonnes dont le départ du fût inférieur, au-dessus de la base, est enveloppé de feuilles d'acanthes, se retrouvent déja sur la colonne aux Danseuses de Delphes, témoignant ainsi de l'influence exercée sur le déror de ces édifices 48 .

Ica découverte de poteries des $\mathrm{IV}^{\mathrm{C}}$ et $\mathrm{V}^{\mathrm{C}}$ sic̀cles permet de dater de ces époques. l'abandon du théâtre de Valence (Drôme) ${ }^{49}$. Au théâtre de Mandeure (Doubs) 50 des travaux de consolidation ont été exécutés à la grande porte : le contrefort moderne a été supprimé et le contrefort aulique dégagé, le niveau ancien retrouvé devant l'entrée, ainsi que le chemin d'accès et, dans la partie haute de l'analomma, un nouveau mur de refend de petit appareil.

Est-ce un nymphée qui a été mis au jour à Cimiez (Alpes-Maritimes), dans lil propriété Garin de Coconato 51? I.es thermes de Saint-Hermentaire it Draguignan (Var), en partic dégagés, furent nccupés du $\mathrm{II}^{\circ}$ au $\mathrm{V}^{\mathrm{c}}$ siècle ${ }^{52}$. L.e problème de l'adduction des eaux n'est pas encore r'ésolu dans ce monument qui s'étend sur deux plans entre les marais et le cours de la Nartuby. R. Louis et R. Dauvergne 53 domnent un exposé des découvertes faites dans l'établissement thermal des Fontaines-Salées à Saint-Père-sous-Vézelay (Yonne).

J. Harmand 54 s'est attaché a discuter la valeur archéologique du mot villa, altaché à tort à tout emplacement où ont été ramasscis des tuiles i robord, des tessons.

('i8) Ibid., p. 60.

(49) Bull. soc. d'archéol. et de statis. Dróme, LXXI, 1951, p. 203.

(50) L. LeraT, Gallia, VIII, 1950, p. 155.

(51) F. BenolT, ibid., p. 180.

(52) Ibid., p. 126-127.

(53) J. CalmetTe et Davin, Les grandes heures de Vezzelay, Paris, 1951.

(54) J. Harmand, Rev. archeol., 1951, 2. p. $155-158$. des débris de décorations murales, à toutes les bâtisses antiques éparpillées dans les champs et trop souvent aussi à des ćtablissements métallurgiques. Il y aurait grand intérêt à distinguer villa et ferme. Mais l'accord est encore loin d'être réalisí. $\Lambda$ quel type de construetion faut-il appliquer le mot villa?

4. Occupation territoriale 't voics romaines. - Ce n'est plus un seul cadastre, dont les fragments ont été découverts ì Orange (Vaucluse), sous l'immeuble de la Banque Marseillaise de Crédit 55 , mais quatre ou cinq cadastres différents ayant pour but de donner la description et l'inventaire des terres publiques de la colonic. Ja date de 77 de notre ìre, fournic par l'inscription monumentale du couronnement de ce document, gravée par orde de Vespasien par son proconsul on Narbonnaise, en fait rentrer la rédaction dans: la longue série des actes de cet mmpereur ayant pour objet la récuperration des terres publiques occupées. Ies autres inseriptions sont encore des inventaires : terres récemment conquisez dans la région des iles; affermage de lots du territoire urbain; liste des emplacements publics et des édifices; taxes pesant sur les usurpaleurs des lerres publiques, aver les inlírôts du retard. Ces cadastres renseignent aussi sur l'ctendue du territoire des 'Tricastins, plus grand qu'on ne le supposait, ct donne certaines indications topographiques sur les fleuves et les cours d'eau, dont lo lracé ne paraît pas avoir varié Hepuis l'époque romaine. Des concordanres qui so manifestent entres la mature du sol actucl ot du sol ancien, d'iprès certaines mentions du cadastre agraire, il résulte que les vétérans de la $I I^{\circ}$ Gallica gardaient les terres fertiles ol, rendaient.

(55) J. SAUTEL, C.R.A.I., 1951, p. 236-238, 242-244; A. Piganiol, ibid., p. 89-90, 244; du même, Ann. Coll. Fr., 1952, p. 230. 
aux indigènes les lerrains peu productifs, marais, garrigues, sols caillouteux.

L'homme apparait tardivement dans lid vallée moyenne de la Seille 56 , nappe forestière entrecoupée de plaines et de clairières. Au Néolithique et pendant les âges du Bronze et du Fer, il s'installe sur les promontoires et les hauteurs dominant la rivière. $\Lambda$ La Tène, le sel y attire les Celtes. Aux temps gallo-romains, de grands domaines sont limités par les routes, disparaissant avec les invasions de la seconde moitić du III $^{\mathrm{e}}$ siècle. Une occupation très dense s'observe ensuite dans la valléc et les défrichements sont importants. Barrés à l'Ouest par les Hautes Vosges, prolongées à l'Est par la plaine, les arrondissements de Thann el de Gucbwiller (Haut-Rhiñ) ne furent pas des centres importants à l'époque romaine. la montagne et la forêt à l'Ouest, la difficulté de la traversée des Vosges, les conditions climatiques furent de sérieux obstacles au pcuplement. Aussi comptet-on peu de découvertes archéologiques importantes: villa de schimmelrain. mansio de Wittelsheim, élablissement dı Burghofen. Le pays ćtait cependant traversé par de grandes routes du Nord au S:ill et du Sud-Est au Nord-Ouest; une autre voie franchissait la montagne, assurant les communications avec la Lorraine 57 . Poursuivant ses études sur l'histoire des pays qui ont formé le département de l'Ain, F. Dubois 58 traite du groupe d'Ambérieu- $\Lambda$ mbronay.

De nombreuses découvertes viennent enrichir la carte archéologique de la Gaule romaine. Dans le Midi, des villas ont ćté signalées aux Baumelles à Saint-

(56) M. Toussuint, B.A.C., 1943-1945, p. $503-545$.

(57) L. G. IVERnER, Rev. d'Alsace, 90, 19501951, p. 16-29.

(58) E. Dubois, Bull. soc. natural. et archéolog. Ain, $\mathrm{n}^{\circ} 65,1951$, p. 57-67.
Cyr-sur-Mer 59 et à Romanin (Bouchesdu-Rhứnc) sur le terrain de vol à voile ${ }^{60}$, l'une et l'autre contemporaines du début de l'Empire. A Tarascon (Bouches-duRhône), le sol de la station Traiectum Rhodani a été retrouvé à trois mètres de profondeur à l'Est de la rue de la République el à une centaine de mètres du pont suspendu 01 : mosaïque en opus signinum attenant à un mur orienté EstOuest, analogue à certains pavements de Glanum et d'Ampurias. Aux Pennes-Mirabeau 62, l'abri sous roche de la Grande Baume a été occupé du milieu du II sièule au début du va. Des sols bćtonnćs ct des réservoirs ont été trouvés dans l'oppidum de Saint-Martin à Mandelieu-I,aNapoule (Alpes-Maritimes) 63, el à Fos (Bouches-du-Rhône) des recherches sousmarines ont révélé l'existence de murs en place dans la vasc, restes d'une habitation effondrée au temps de Tibèrc (tessons d'Arezio et de La Graufesenque, lampe cn forme de navire et Siròne avec pêcheur) ${ }^{64}$. En Camarguc, sous la butle de la "Terre du Cimetièrc ", à Cabane 65, au Mas dc Catane près du Rhône et de SaintFerréol, s'étendent les ruines d'un établissement agricole de la fin du nII siècle qui a donné une statuc de Priape. Un domaine rural de haute époque, au "T'rou d'Or" de Mascapien à Tourves (Var) était doté d'un important équipement agricole : pressoir d'huilerie et bassins bélonnés pour l'huile et le vin; four circulaire à sol bétonné dans une construction carrée de pelit appareil, avec canalisations verticales communiquant

(59) F. BexoIt, Gallia, VIII, 1950, p. 12\%128.

(60) Ibid., p. 124.

(61) Ibid., p. 124.

(62) Ibid., p. 123-124.

(63) Ibid., p. 130.

(64) Ibid., p. 123.

(65) Ibid., p. 122 
avec un système de canaux de briques 66 . Dans le Vaucluse, à Puymérass 67 et à Visan 68 des exploitations rurales des $\mathrm{I}^{\mathrm{er}}$, $\mathrm{III}^{\mathrm{e}}$ et $I V^{\circ}$ siècles ont été reconnues. Le creusemenl de la dérivation du Rhòne, entre Donzère et Mondragon 69, dont le Iracé correspond à celui de la voie d'Agrippa d'Arles a Lyon, a mis au jour des ruines à Saint-Paul-de-Sénoset à La Martinière. $\Lambda$ Gordes ${ }^{70}$, au Sud-Est du hameau des Gros, et à La Villeneuve un site important, occupé d'Auguste à Gallien, mériterait. d'être fouillé.

On constate une semblable dispersion de l'habitat sur le territoire de l'Aude: station à Cournanel $\mathbf{7 1}$ sur une petite plateforme de la colline à laquelle s'adosse le village actuel, et dont la surface a été aplanie, succédant à un établissement des $V^{*}-I I^{n}$ siècles; villas dans la campagne de la haute vallée de la riviòre qui a participé à la civilisation des oppida du Ianguedor méditerranćen et a été peuplée à l'époque romaine; exploitation agricole au Poulignan de Trausse 72 à Montanié, dans les vallées de la Besse et du Barrou ${ }^{\mathbf{7 3}}$, à Capendu avec vestiges préromains ${ }^{74}$, de même qu'à Mairac ${ }^{75}$; villa à Isa Tasque à Cadeilhan-Saint-Clar 76; nouvelle mosaïque à Pompogne 77 , mais on nublie d'indiquer le sujet; briques au tertre de Fronsac (Gironde) ${ }^{178}$; ruines entre Varacre,

(66) Ibid., p. 128-129.

(67) J. Sautel, ibid., p. 136.

(68) Ibid., p. 136.

(69) Ibid., p. 138.

(70) Ibid., p. 138-139.

(71) R. Lizop, Bull. soc. et. scientif. Aude, LI, 1950, p. 35-43.

(72) H. Taldavignes et J. Arnal, Rev. d'ét. ligures, XVI, 1950, p. 246.

(73) Bull. soc. ét. scientif. Aude, LI, 1950, p. $\mathrm{xv}$.

(74) Ibid., p. 33-34.

(75) Ibid., p. XVIII-XIX.

(76) Ann. Midi, 63, 1951, p. 281.

(77) Rev. Agenais, 77, 1951, p. 55-57.

(78) B. Ducasse, Rer. hist. et archeol. Libournais, 1951, p. 3.
Saillac et Bach (Lot) où pourrait être recherché l'emplacement de Varadetum, auprès de la voie d'Auvergne en Gascogne, que fréquentèrent les pèlerins de Compostelle 79 , près de l'église de Cany-Luzech (Lot) ${ }^{80}$; tessons à Arzenc d'Apcher (Lozère) ${ }^{81}$; haut-lieu avec fanum de Mercure, déjà connu à Toul-Saint-Croix (Corrìze) ${ }^{82}$; mosaïques et ruines sur une dizaine d'hectares à Fonduıure (Charente) quartier Nord de Villard 83 ; slatuette do femme assise posée sur un petit autel à Angoulême au bas de la rue Jules-Ferry. près de l'église de Saint-Martin où l'on décourrit autrefois des bases de colonnes 84; habitat au Pouget, commune de Sainte-Innocence 85 et centre agricole et métallurgique à Puydivert., commune de Bourdeix (Dordogne) 86 .

Dans le Maine et en Normandie, quelques découvertes sont à signaler à Saulges. (Mayenne), sur la face Nor(l-()uest du plateau de Dame ${ }^{87}$; au Sul de Barfleur (Manche) sur la tranche d'uno falaise bordant la plage de sable 88; à Canny et ì Frénouville (Calvados) ${ }^{89}$, et pour la première fois à Caen où on recucillit, houlevard des Alliés, un fragment de verre gallo-romain ${ }^{90}$. I! parait difficile de re-

(79) Prat, Bull. soc. d'et. Lot, 1951, p. 108109.

(80) Desprats, ibid., p. 116.

(81) Bull. trim. soc. sc., lettr. et arts Lozire, 1951, p. 108.

(82) Dr. G. Janicaud, Bull. soc. emul. Bourbonnais, 1951, p. 74.

(83) Bull. mens. soc. hist. et archeol. Cha-

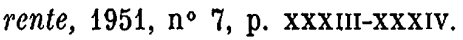

(84) Ibid., p. XXXVIII-XXXIX.

(85) E. VauTier, Bull. soc. hist. et archéol. perrigord, LXXXVIII, 1951, p. 117.

(86) C. Barrière, B.S.P.F., XLVIII, 1951, p. 154-157.

(87) Le Pays du Maine, 2e sér., XXXI, 1951. p. 117.

(88) R. MESLIN, Bull. soc. antiq. Normandie, LI, 1948-1951, p. 466.

(89) B. Martin, ibid., p. 357.

(90) Monutiluten, ibid., p. 351. 
connaître l'exislence d'un fundus antique dans la région de Saint-Pierrc-du-Breuil près de Mézidun (Calvados) 01.

L'enceinte de Beaurais (Oise), disparuc dans sa partie méridionalc, a pu être suivie à l'Ouest dans le Jardin du Palais de Justice, où clle est flanquéc de tours scmicirculaires $9 \%$.

I)ans l'Ardèche des emplacements d'habitations ont été reconnus au pouzin, sur la rive droite du Rhône, au quartier de la ('endarmeric ${ }^{83}$; à Toulaud, dans le quarlier des Fonds, murs de soutènement des cultures d'une villa ${ }^{94}$; à Crussols, restes dun village occupé avant la comquête, situé sur un plateau à un kilomètre au Sud du châleau ${ }^{95}$; à Saint-Jean-de-Muzols, au quartier Saint-Fstève, substructions et grand mur de quai près du Rhône ${ }^{96}$. Dans la Drobme: exploitation agricole sur un petit platean au quarticr du collet d'Aouste ${ }^{27}$; mosaïque au nouveau groups. scolaire avec rose des vents noirc et blanche et tuiles à rebord près de la voie ferrée, au centre de l'agglomération romaine de Saint-Paul-Trois-Châteaux ${ }^{98}$; tombes et tuiles aux environs de Die, à Aix-en-Diois, Aurel, Pont-de-Barrit. Ponlcx, Epic ${ }^{99}$. Une zone de peuplement aŝsez importante couvre la région de Buisles-Baronnies 100; constructions sur le plateau de Saint-Jean, une villa sur les bords de l'Ouvèze ef de nombrcuses sépultures.

Dans l'Tsère, Bourgoin parait avoir été lc siège d'une station routière sur la

(91) Nbbé Tesson, ibid., p. 91.

(92) Comm. des fouilles, 27 mai 1952.

(93) J. SAUTEL, Gallia, VIII, 1950, p. 133.

(94) Ibid., p. 133-134.

(95) Ibid., p. 134.

(96) Ibid., p. 134.

(97) Ibid., p. 135.

(98) Du MÊMrs, ibid., p. 136; Bull. soc. d'urchéol. et de statis. Drôme, LXXI, 1951, p. 20:3.

(99) J. SAUtel, Gallia, VIII, 1950, p. 235.

(100) Dr. G. BERNARD, Bull. soc. d'archéol. et de statis. Drome, LXI, 1951, p. 205. roule d'Italie 101 et dans le Doubs, à Bcsançon, les fouilles dans le terrain acquis par l'Université à la Banque de France ont dégagé un établissement ave hypocauste ${ }^{102}$.

De nouvelles salles ont été déblayces dans la villa de Sérainville (Meurthe-etMoselle), détruite dans le troisiòme quart du Iy!e siècle 103. Desservi par une voie romainc, le territoirc situé sur la rive. droite de la Nied allenande, entre Crihange et Elvange, a fait connaitre des vestiges gallo-romains sur le ban de la fermo de Mouzaïa, au Ham et à Flétrange 104. la villa qui se dresse à la limite d'Einvaux et de Clayeures était le centre d'un domaine important, autour duquel se groupent de nombreuses ruines et les lombes d'un cimetièr'e mérovingien. In autre foyer important est placé au village de Chaumont 105. A quelques centaines de mètres au-dessous du col de Saverne, entre la route actuelle el le tracé de la voie romaine, une construction antique, au lieu Usspann 106, correspond aux ruines d'unc station routière, bâtiment reclangulairc avec partie en saillic sous auvent et traces de scellements d'attache pour les chevaux, ćcurie ou caravansćrail, incendié à plusieurs reprises, en 70 et 97 , lors des événements qui amenèrent la destruction du camp de Strashourg. Des outils de fer, des fibules de bronze, des monnaies d'Auguste, de Tibère, de Nóron et de Domitien, des tessons de Ta Graufesenque, de Chémery (Satto) et de Rheinzabern, datent ce gîte d'étapes de la premièrc moitié du rer sir̀cle de notre ère.

(101) P. Wuilleumier, Gallia, VIlI, 1950, p. 145.

(102) L. Lerat, ibid., p. 155.

(103) Le pays lorrain, 32, 1951, p. 94.

(104) Ibid., p. 7.

(105) M. Toussaint, B.A.C., 1943-1945, p. 578-579.

(106) J.-J. HaTT, Gallia, VIII, 1950, p. 166; Rev. archéol. Est, II, 1951, p. 121. 
Au gué d'Aynard, ver's les limites des communes de Bonnay et de Cortevaulx (Saône-ct-Joire), au lieu dit "I.es Terres Saint-Germain "107, pròs de la voie pavéc traversant la Guyc, à une villa gallo-romaine se superposent un établissement chrítien et des sépultures.

I.a borue milliaire de Treilles, seul milliaire connu antérieurement à l'époque impériale, a été l'objet d'une rícente étude 108 : il est le témoin d'un bornage conLemporain du séjour en Gaule de Domilius henobarbus, qualifié ici d'imperator. l.e milliaire est postérieur à la victoire de Fabius Maximus et de Domitius sur la coalition des Allobroges et des Arvernes, dans la vallée du Rhòne, le 18 août 121, contemporain de la fondation de Narbonne cn 118, qui représente la date minimum vraisemblable pour son érection, à $\mathrm{xx}$ milles de Narbonne, tout près d'un guć. confirmant ainsi le passage de la voie primitive dans la plaine littorale, alors que l'on ne connaissait encore que le tracé d'époque impériale sur les Corbières, dominant la plaine à l'Ouest. Entre le Rhône et le Vidourle, l'examen des milliaires prouve que la voie Domitienne ne passait pas par la faille de Roquepartide, qui n'est qu'un chemin muletier, un raccourci. La route évitait aussi la dépression marécageuse du viaduc ${ }^{109}$.

La plaque de terre-cuite découverte à Macquenoise 110, d'après les noms des stations et la nature des représentations, reproduirait (?) un original antique qu'n aurait voulu remplacer. Tse monument, trouvé en 1947, à en juger par ses caractires épigraphiques et le tracé de sa cartc.

(10i) L. Armand-Caillat, Rev, archeol. Est, II, 1951, p. 61-62; P. WUILleUMier, Gallia, VIII, 1950, p. 151-153.

(108) J. Campardou, Gallia, VII, 2, 1949 p. 19 (6207); P.-M. DuVaL, ibid., p. 207-231. DU MÊME, C.R.A.I., 1951, p. 161-165.

(109) M. LouIs, B.A.C., 1943-1945, p. 547578. est de facture moderne, mais reposerait sur un document antique pour le fond: exactitude dans l'orthographo des noms des stations et dans l'ordre oì elles sont inumérćes, ressemblances des édicules figurés sur la carte avec des constructions analogues, relais ou sanctuaires attestés dans l'antiquité. Ia plarue n'est pas sans offrir encore des rapporls avec les tahlettes d'Astorga. Les indications qu'elle fournit se rapportent au trajet de Vervins à Aix-en-Provence, route suivie par les voiluriers pour atteindre la Méditerranée. On remarquera que la pièce a été découverte au voisinage d'un sanctuaire de frontic̀re et d'une verrerie gallo-l'omaine et mérovingienne utilisant la soude mirlilerranéenne.

Des conditions de la circulation par les voies de communication entre les pays de la Méditerrance et ceux de l'Ntlantigue. pendant le Moyen âge 111, il est possible de retirer des enseignements sur les grandes routes du commerce antique. LiAtlantique représente alors la fin du monde connu. De là vient l'étain des Cassitérides. I'extension de la civilisition à l'époque romaine conduit les populations de l'Atlantique à désirer les épicos, les tissus précieux, les hijoux de l'Orient méditerranéen; et l'unification apportée par Rome, puis par le Christianisme, déterminera des courants plus intenses des hommes et des marchandises, qui preildront la grande route naturelle do la Míditerranéc à la Mer du Nord, par los val-

(110) J. Vannérus, Bull. classe lettr., sc. mor. et polit. Acad. roy. Belgique, 1951, p. 468498. Dans un article de la Rev. archéol. list., III. 1952, p. 43-51, P. LEBEL conteste l'authenlicité de la carte routière, où il retrouve une combinaison de la Table de Peutinger et de l'Itinéraire d'Antonin, l'utilisation des cartes de Desjardins, et conclut à un faux, ce que préciserait encore l'absence de distances, de dédicace, de trous de suspension.

(111) Y. Revouard, Mél. L. Halphen, p. 387394 
lées du Rhône, de la Saone, de la Seine et par les plaines de Champagne, sans compter les chemins plus occidentaux reliant directement les deux bassins. I'un d'entre eux, entre le Massif Central et les Pyrénées, par la vallée de l'Aude, le seuil de Naurouze et la vallćc de la Garonne, est une de ces voies naturelles facilos entre Méditerranée- $\Lambda$ tlantique et pays septentrionaux. Connu depuis Strabon, il a été largement suivi à l'époque romaine. r'absence d'utriculaires montre que la Garonne était moins fréquentée. On notera que la voie romaine évite souvent le Heuve, dont les crues ruinent les chaussćes, et coupe plus profondément à travers la Gascogne, do Bazas à Toulouse, par Fauze et Auch, et d'Agen à Toulouse par L.cctoure. Ia section 'Toulouse-Carcassonne-Narbonne est une voie de passagc obligće entre deux chaînes de montagne ct utilisée par tous les voyageurs. Au Nord ou au Sud, ce sont les routes subpyrénéennes de Toulouse à Saint-Bertrand-dc-Comminges, Dax et Bayonne, à travers le Béarn; de Barcelone, par la vallée de l'shre, un autre chemin franchit les Cantabres pour aboutir aux ports des côtes basque el asturienne.

Depuis longtemps on cherche une explication à toutes les crreurs concernant la longueur des étapes au sortir de T.yon 112. Celles-ci auraient pour origine une faute de transcripteur, le chiffre Xvi représentant la distance de IyonTudna aurait été malencontreusement reporté le long du trait figurant la longueur de l'étape Lyon-Vicnne, lequel est de xvir milles romains.

Un document apporté par une transaction de 1523, entre la communauté de la ville de Mazan (Vaucluse) ct les seigneurs de la dite ville, intéresse les vieux chemins d'un territoire traversé et cotové

(112) A. Aunis, Ree. archéol. Est., II, 1951, p. $97-104$. par des ruisseaux descendant du Ventoux ou de la chaîne de Vaucluse, au centre du Comtat, à l'Est de Carpentras et au débouché du passage entre les vallées du Rhône et de la Durance, à mi-route de Cavaillon et de Vaison, presque isolé au milieu d'un pays accidenté et boisć $\mathbf{1 1 3}$. Jes précisions sont données au tracé de la voie romaine Lutetia-Rotomagus dans le Parisis et le Vexin français, sur le tronçon Paris - Saint-Clair-sur-Epte ${ }^{114}$. Une grande route de frontière traverso la vallée de l'Allaine à la hauteur de Delle 115 et, à Vézelois, à scpt kilomètres au Sud-Est de Belfort, au lieu dit "Isa Rouge Vie " et à "Petite Vie" de Chévremont, lieu dit "Le Tora". on a retrouvó le tracé, large de cinq mètres, de la route de Mandeure à Strashourg 116. Sur cette môme route de Mandeure, au cours de travaux d'aménagement du deuxième tronçon du canal d'Alsace, des gués et des chemins en caillebotis ont été relevés près de l'ancien lit du Rhin, puis entre Kembs et Kleinkembs, et dans l'are de la courbo donnant accès sur le rebord du plateau, face à la rive badoise, des vestiges de constructions : piles en blocage et masses de maçonnerie, localisées autour de certains points d'émergence, appartenant à un pont ruiné par de fortes inondations et dépouillé de ses grosses pierres par les riverains, fondé sur un banc de mollasse assez dur à la fin du rer siècle de notre ère et longtemps utilisé 117. Kembs, placé à l'extrémité de la voie romaine de Besancon, la grande route de pénétration vers les Champs Décumates, était au IV s. une

(113) L. Carrias, B.A.C., 1943-1945, p. 296297.

(114) J. Toutain, ibid., p. 267-279.

(115) J. JoACHM, Bull. soc. belfortaine émul. 1950-1951, p. 16-33.

(116) R. BERMON, ibid., p. 59-63.

(117) J.-J. HATT, Rev. archéol. Est, II, 1951. p. 120-121; 4te rapp. soc. suisse de préhist., 1951, p. 134; Gallia, VIII, 1950, p. 166-168. 
importante forteresse pourvue d'une têlc de pont sur la rive badoise. Par sa situatlion stratégique Kembs protégreait la route menant au seuil de Bourgogne, à la vallée du Rhône et à l'Italic. C'était, au temps de Valentinien, l'un des verrous du système défensif sur le Rhin supérieur.

5. Les sépultures. - Une excellente thèse de doctorat traite de la tombe galloromaine 118 , en tant que fait social permettant de rechercher en profondeur " les effets de la romanisation dans la masse des populations gallo-romaines ". C'est aussi un essai de synthèse de ce qu'on peut savoir de l'art funéraire, des grandes lignes de son évolution et de ses rapports avec les conditions sociales el éronomiques du moment. Dans cette enquête une très large part est faite à la notion de quantité en utilisant le pro"édé de la statistique, et au facteur chronologique, cn recherchant tous les moyens dont on peut disposer pour classer dans le temps inscriptions et monuments figurés. Comme on le constate de plus en plus, il n'y cut pas rupture entre les coutumes funéraires de la t'ène et celles des temps de la domination romaine. Tonomastique celtique ne disparait pas et sa rípartition révèle, au $\mathrm{II}^{\circ}$ siècle, un exode des campagnes vers les villes à la faveur du développement industriel et commercial, et la persistance dans la Gaule centrale. le Comminges, le Couscrans, les Basses Vosges, d'un particularisme local se traduisant aussi par le monument funéraire. In autre aspect de la constitution et des tondances de la société se dégage de l'examen des noms à caractère religieux, tómoins de la pénétration des idées du svn-

(118) J.-J. HATT, La tombe gallo-romaine. Recherches sur les inscriptions et les monuments funeraires gallo-romains des trois premiers siecles de notre eve, Paris, Les Presses Universitaires de France, 1951. Cf. R. LANTIER, Journ. Sav., 1951, p. 157-170. crétisme gréco-oriental dans les masses populaires. L'apport romain en matière lunérairc ne représente, là encore, qu'un déguisement momentané et la nouvelle socićté est loin d'avoir oublié son passé celtique. Il en est de même de l'art funćraire, dont les manifestations peuvent s'ordonner en deux périodes bien tranchées : au Ier siècle, en Narbonnaise et en Germanie, une sculpture sépulcrale d'inspiration et de caractere essentiellement romains; aux $\mathrm{II}^{\mathrm{e}}$ et $\mathrm{III}^{\circ}$ siècles, un art filnéraire régional ayant son originalité propre. A chaque région correspond un milieu particulier et l'expansion de certaines formes, le grand mausolée par exemple, a été favorisée par la formation d'une aristocratie foncière dans les grands domaines aquitains et l'avènement d'une classe de riches nígociants, installés au carrefour des voies vers la Germanie, principalement dansi la valléc de la Moselle. La "route du vin" de Trèves à Bordeaux, la "roule du drap" par les vallées du Rhòne, de la saòne of de la Moselle, sont jalonnées par ces grands monuments. A l'unificalion du type monumental correspond l'unité du décor sculptural, portrait des défunts, représentations empruntées à la vie quolidienne, aux scènes mythologiques. I’une des caractéristiques de celle plastique sera, à partir des Antonins, lo díveloppement du naturalisme, aussi hien dans le portrait, par l'ohservation du modìle vivant, l'itude du costume local, que dans les épisodes de hesognes journalières, et III thème symbolique, le Repas funèbre. deviendra une scène de genre, Jo "Repas des Paysanz ". Dans le cadre ehronologique, cet art funéraire s'inserit en quatre phases successives depuis le commencement du II siòcle jusqu'au dernier liers du IIr. Une grande variété se manireste dans la forme du monument sépulrral, tantot d'influenere ronfaine, tantôs de caractère régional. 
Des sépultures du $11^{e}$ siècle, sous lauses, ont élé trouvées à Vitrolles 119 el à Eygalières (Bouches - du - Rhơne) ${ }^{120}$; à couvercle de tuiles en bâtière sur la butte de Saint-Cassieno à Cannes (AlpesMaritimes) ${ }^{121}$; des épilaphes à Valréas, a Robion 122, au Crestel-et-Gargas (Vaucluse) ${ }^{123}$; des incinérations du $\mathrm{I}^{\mathrm{e}}$ siòcle dans la cour du lycée Jules-Ferry à Tournon (Ardèche), à l'intérieur de deux amphores fusclées ${ }^{124}$.

A Vichy (Allier) 125, lo cimctière déja connu du jardin de l'Hôpital Mililaire, a donné deux nouvelles sépultures du II" siècle, avec vases à relicfs d'applicjue, masques de Pan et de Fleuves à reflets métalliques, peut-être de fabrication locale, des assiettes à trois pieds et de grandes ollae. Au Bouy (Puy-de-Dôme), dans un mur de l'ancien château. me inscription funérairc sur hloc de granulite, présente un certain nombre de particularités: moniminto, souvenir d'une graphie archaïque et d'une prononciation locale; à la fin de la troisième ligne un () pointé indique que lo personnage dont le nom précède était mort ${ }^{126}$. Toes incinórations dans des coffres de granil sont signalécs à Dougicr. commune de SaintAignan-près-Groc (Creuse) avec urne cinéraire en verre blcu, clous et trépied de fer 127, au Charlat, cne d'Tssel (Corrèzo). du début de l'Empire 128, à Ta Churlerie,

(119) F. BenoIt, Gallia, VIII, 1950, p. 124. (120) Ibid., p. 124.

(121) Ibid., p. 130.

(122) J. SAuTeL, ibid., p. 136-138.

(123) Ibid., p. 138.

(124) F. BENoTT, ibid., p. 133.

(125) Dr. A. MORLET, Plages et villes d'eaux, no 24, juillet 1951, p. 40-41.

(126) J. VeNDRYES, C.R.A.I., 1951, p. 131134; Bull. soc. hist. et scientif. Auvergne, 1951, p. 68-69.

(127) Dr G. JantCaud, B.A.C., 1943-1945. p. 170-171.

(128) M. VazeItufs, Gallia. VIII, 1950, p. 104-106. che de Saint-Symphorien, appartenanl au Ir siècle ${ }^{\mathbf{r}}$, au Lavon à Montmorillon (Vienne) ${ }^{130}$. Le tumulus des Tourettes, à Châtcau-Ponsac (Haute-Vienne), abritait une chambre en maçonnerie, couverte d'une rangée de pierres plates, contenant de nombreux charbons, un tesson de vase en lerre noire et trois en lerre rouge 131 .

Le cimetière de la carricire de Buss'o:l à Étrechy (Cher), sur la route de Jalognes ${ }^{132}$, a donné vingt-six sepulture: pratiquées dans le rocher et deux dinls des sarcophages. Les mobiliers, caractérisés par l'abondance des vases, dont un gobelet cn verrc aux scrpents et une polerie avec arbre stylisć et dédicace à Mercure, comprennent aussi des bagues, des boucles d'oreilles et des monnaies des $\mathrm{III}^{\mathrm{e}}$ et $I V^{\circ}$ siècles. I,es offrandes alimentaires sont représentécs par des abats de jeunes mammifìres et des volailles. I a présence de clous marque l'emploi des cercueils. lin autre cimetière qui arait déjà donné des sarcophages de calcaire slriés en arêtes de poisson, s'ćtend le long du chemin vicinal de Pessac à Nergis, à Tréfontaine (Ioiret) ${ }^{133}$. Dans les huit nouvelles tombes, surmontécs vers les pieds d'une slèle, les squelcttes étaient couchés sur le ventre en pleine terre, un vase aux pieds (rve- $\mathrm{V}^{\mathrm{c}}$ siècles).

In nouvcau sarcophage avec ascia et scie, a ćté découvert à Rouen (SeineInféricure), en fr"e du $n " 72$ de la rue du Renard, sur le iraec de la voic RouenJillebonne et l'emplacement d'un cime-

(129) J. PERRIER, Bull. soc. urchéol. et hist. Limousin, L.XXXIII, 1951, p. 371.

(130) F. Eygun, Bull. soc. antiq. Ouest, $4^{\circ}$ sér., J, 1951, p. 809.

(131) M. BAURÉROT, B.S.P.F., XLVIJI, 1951, p. $538-540$.

(132) P. Cravayat, Mém. union soc. sav. Bourges, II, 19:4-1950, p. 7-14; R. Tours, Gallia, VIII, 1950, p. 172-175.

(133) 13. Iovts, Gallia, VIII, 1950. p. 172. 
tière situé place Ciauchoise, sous l'église Saint-Gervais et s'étendant sous les rues transversales 134 .

Près d'habitations gallo-romaines, rue du Fossé et rue de Saint-6mer, à Thérouanne (Pas-de-Calais), une tombe à inhumation dans un cercueil était accompagnée de tessons et d'un plomb conique à pointe arrondie $\mathbf{1 3 5}$.

Lors de la construction d'un garage a Lyyon ${ }^{136}$, entre le $n^{\circ} 10$ de la rue des Fantasques et le nur de soutènement de la rue de Magneval, à l'angle de deux gros murs, on mit au jour le squelette d'un soldat accompagné de monnaies, jadis contenues dans une ceinture, grand bronze de Lucile, douze deniers d'argent de Marc-Antoine (33-31), de Vespasien, de Domitien, de 'Trajan, d'Antonin, de Faustine la Jeune, de Commode et de Septime-Sévère, et de pièces d'équipement, épée à garde de cuivre, bouterolle, fibule en forme de chien, patte ouvragée et aiguillettes de baudrier, boucle $\dot{a}$ anneau, bouton à double rondelle, neuf lettres de bronze découpé de l'inseription vtere felix Alae..., premier exemple de caractères fixés à un bouclier. Un deniet. à fleur de coin, frappé en 194, indique que ce militaire périt dans la grande bataille de 197, opposant Albin à ScptimleSévère. Or, le site de la bataille n'biait, pas encore fixé de façon précise, la déconverte du plateau de saint-Glair, prolongeant le plateau de Rillieux, vient à l'appui de la thèse plaçant le combat sur le plateau de Rillieux. Au quartier de Vaisse, 45, rue du Tunnel. la découverte d'épitaphes et d'une urne cinéraire montre que le rimetière repéré sous l'église

(134) Ibid., p. 236-239; VII, 2, 1949, p. 236. (135) Bull. trim. soc. acad. antiq. Morinie, XVII, 1951, p. 476-478.

(136) P. Wulldeumier, Gallia, VIII, 1950. p. $146-150$
Saint-Pierre, s'étend plus loin vers le Sud, le long de la voie franchissant l'enceinte sur le plateau de la sarra (Ir $\left.{ }^{e} \mathrm{~S}.\right)$. A Vicnne (Isc̀re) ${ }^{137}$, lor's de la construition de l'Hôtel des postes, sur l'ancien cimetière de Saint-Gervais, au sud de la gare, une épitaphe mentionno un tabularius publicus ( $\mathrm{II}^{\mathrm{e}}$ ou $\mathrm{III}^{\mathrm{e}}$ s.).

Un abri sous roche à la puinte méridionale d'Alise-Sainte-Reine (Còte-d'Or), à la "Croix Saint-Charles ": abritait un cimetiòre de bébés 138 , à la base d'une couche contemporaine des rignes de Claude et de Néron. Jes corps a avaient été placés dans de petits cercueils de bois, certains dans un loculus de pierres fermé par une dalle, et près des squelettes on recueillit quelques petits vases et des fibules.

Dans l'Est de la Gaule, à Mondelange (Moselle), un cimetière du $\mathrm{IV}^{\mathrm{e}}$ sic̀cle de notre ìre s'étend en bordure de la route nationale $n^{\circ} 53$ de Metz à 'Thionville 139. A Gosselming (Moselle), près de la ferme d'Alzing, quatre sépultures i incineration sont contemporaines de la seconde moilié du I $^{\text {er }}$ siècle aprìs J.-C.140.

6. Arts et Méliers. Commeree' el Industrie. - Aux abords de ce même cimetière d'Alzing travaillait un atelier spérialisé dans la fabrication des urnes funéraires, exécutées en deux parties, récipient et couvercle, dans un bloc de gres vosgien 141. Il devait exister ì Vertaul! (Cite-d'Or), un petit contre industriel de coutelliers 142. Leur's productions sont représentées par des couteaux de fer à

(137) Ibid., p. 145

(138) L. LERAT, ibid., p. 155-158; J. JOLY, Rev. archéol. Est, II, 1951, p. 119-121.

(139) E. Delont, Mém. acad. nat. Metz, n. s. I. 1951 , p. 80-82.

(140) Ibid., p. 9

(141) Ibid., p. 11-12.

(142) R. PARIS, Bull. soc. hist. et archeol. Châtillonais, 1949-1950, p. 29-31. 
lame fixe ou mobile et à manche d'os à décor le plus souvent géométrique, parfois animalier (chien ou sanglier).

Fin 1947 et 1948 , dans les sables des berges de la Suône, on recuellit un certain nombre de vaissolles de bronze ${ }^{143}$ : près du porl d'Ouroux, patère à manche ajouré accosté de deux têtes de cygnes gravées de type havien; près du gué d'Allériot, au Nord de Chalon-sur-Saòne, deux casseroles avec thyrse gravé sur le manche et portant la marque de Florus. Cette dernière trouvaille, rapprochée de la masse de documents semblables trouvés à Chalon dans le lil de la Saine, posc le problc̀me de la localisation de l'alelier de Florus dans cette ville. Ia ville pourrait avoir été par son. port un centre de diffusion des orfevreries et des bronzes d'origine méridionale, acheminés par la Saòne, mais aussi un contre de fabrication pour les chaudronneries et les statuettos de bronze si nombreuses dans le Chalonnais. La clé de bronze, dont le manche se termine par une main tenant une boule de fard, est un modèle rógional. Enfin l'originalité des objets de parure damasquinés, localisés entre Charnay-les-Chalon et Tournus, laisse entreroir l'utilisation par les Mérovingiens d'une main-d'ouvre gallo-romaine locale. ancienne et expérimentée.

Une exploitation de fours à chaux, contemporaine des Antonins, était située ì Saint-Martin-du-Tertre (Yonne), sur le versant du vallon des Glaciers, à gauche de la route de Vaulx, un peu en avant d'une petite chapclle 144. I'rès des vestiges de hangars à trous de poteaux, trois puits avaient été creuscis dans la craie et deux murettes disposées en angle aigu extérieurement au banc de craic, ćtaient

(143) L. Armand-Catrtat, Gallia, VIII, 1950, p. 233-235.

(144) J: CONDRAY, Rev. archéol, Est, II, 1951, p. 196-199. calées par un matelas dargile rouge vitrifiée. Un ressaut à $1 \mathrm{~m}, 10 \mathrm{du}$ fond sert de point d'appui à un plancher de grosses branches soutenant la masse des malériaux destinés à la cuisson au-dessus de la chambre de combustion pour la misc à feu. A la partic inférieure, une ouverture permettait l'extraction de la chaux. Chaque four n'a été utilisć qu'unc seule fois. Peut-être les prétendus silos de Michery sont-ils aussi des fours à chaux.

Près de Peyrelevade (Lozère), à cinq conts mètres des grottes préhistoriques 145, un carrier avait installe son chantier, à Pélis, pour la fabrication d'éléments architecturaux et de meules. $A$ la question: les Romains ont-ils transporté la pierre d'Arles, c'est-à-dire de Fontvieillc (Bouches-du-Rhône), à Toulouse? on peut répondre par la négative ${ }^{146}$.

Une usine de conserves de poissons, en activité au rer siècle de notre ère, bassins carrés alignés, analogues à ccux du Taccarès et de Bétique, a ótú découverte : vitrolles (Bouches-du-Rhône), sur une butte voisine de l'ancien étang du Lion. sur la rive orientale de l'étang do Berre ${ }^{\mathbf{1 4 7}}$.

Jes fouilles du chanoine Ilemet ì La Graufesenque (Aveyron) n'avaient été qu'une suile de sondages dans une plaine s'ćtendant sur une quinzaine d'lectares et ne portèrent que sur la recherche d'un matériel céramique, nógligeant presque entièrement le contexte archíologique. Une exploration plus móthodique du site s'imposait pour préciser aussi bien les problèmes relatifs aux origines de colle industrie et de sa dispersion que de son organisation et de la condition ouvrière

(145) Desprest, Bull. soc. d'ét. Lot, 1951. p. 151.

(146) Ann. Midi, 63, 1951, p. 71-74.

(147) F. BenoIt, Gallia, VIII, 1950, p. 124. 
des potiers. Pour y repondre, le regrettí A. Albenque et L. Balisan 148 ont entrepris dója deux campagnes à la Graufesenque, sur un emplacement situé ì soixante mitres au sud-Sud-Fst de la ferme de Ja Graufesenque, sur la propriété de M. Miquel, à égale distance entre les sites fouillés par les abbés Cérès et Ilermet, entre la Dourhie et les premières pentes du Iarzac de l'autre. Dans la partie Nord de la tranchée s'étendent les restes d'un habitat gallo-romain, vraisemblablement de basse époque. Dans la région méridionale, la découverte de vestiges d'architecture, fît: do colonnes, bases moulurces en grès, dallages, marque l'emplacement d'un édifice d'une pertaine importance. Au-dessus du dallage. un amas de décombres paraît correspondre au dépotoir d'un atclier de potier : monnaie d'Antonin le Pieux de l'année 137, nombreux tessons de poterie rouge sigillée, outils de potier, supports de vases, cale de four, déhris de tournette et. pour la première fois à $J_{d a}$ Graufesenque. un poinçon-matrice en terre-cuite portant l'image d'un lion accroupi, enfin douze fragments de graffites dont quatre appartiennent à un même compte. T'intérêt principal de ces nouvelles recherches a été de faire connaitre l'existence, sous le niveau gallo-romain, d'un lıorizon próromain représenté par un cimetière à incinćration de La Tène II. apportant aimsi la preuve que le confluent du Tarn et de Ia Dourbie avait été orcupé dès lo Je siècle avant l'ère. Certaines piòces du mobilier funéraire, amphores italiques. poteries campaniennes du $\mathrm{rr}^{\mathrm{e}}$ siècle à díror de palmettes, vases de fahrication indigène, monnaies de Marseille, témoi-

(148) J. VeNDryes, C.R.A.I., 1951, p. 420; R. LaNtier, ibid., p. 420-422; $\Lambda$. ALBENQUe, R.K..., LIII, 1951, p. 71-81; DU MEME, Rev. archéol., 1951, p. 174-190; Rer. Rouergue, V, 1951, p. 45-68; L. BalzAN, ibid., p. 531-533; De Mr̂ur, Gallia, VIII, 1950, p. 174-190. gnent de relations commerciales avec la Héditerranée. Au point de vue de l'hisloire des industries céramiques, ces fouilles apportent les premiers résultats d'une enquête nouvelle. I'existence d'un habitat antérieur à la conquête étant définitivement citablie, ainsi que la présence de poteries de fabrication locale, il est désormais permis de reahereher dans ces modestes fabrications les origines de la grande industrie céramique de Ja (iraufesenque et cela dis la fin du dernier siccle avant notre ìre. Cela est d'autant plus vraisemblable que les l'ícentes découvertes faites à I,ezoux (P'uy-deDome) ont fait connaitre la superposilion directe d'officines gallo-romaines à des ateliers de potiers celtiques. Ises précisions sont également apportées à l'histoire des derniers temps de rette industrie. Ia présence dans le dépotoir de tessons contemporains du rigne d'Antonin le pieux recule bien au delà de l'époque de Vespasien les ultimes manifestations de cette activité : certains ateliers de Ia Graufesenque devaient encore travailler au milieu du re siècle après J.-C. T.e dépotoir pourrait être celui de l'officine de Vitalis, dont on a recueilli plusieurs estampilles : 70 marques, 36 noms de potiers, dont six nouveaux, tous, a l'exceplion de Cintusmus et de Crururo. d'origrine latine, d'où progrìs de la romanisafion. J'artivite reste grande dans ces ateliers du $\mathrm{II}^{\mathrm{e}}$ siècle, puisque rotte officine pouvait assurer la production de plus de 11.000 vases d'apròs l'un des nouveaux comptes. ce qui implique la conservation de déhouchés ef la possibilité d'érouler les marchandises au moins sur des marchés locaux ou régionaux. On peut alors envisager de rapporter à Ta Graufezenque un certain nombre de productions jusqu'alors rattachées à des officines de la Gaule orientale. La romanisation semhle bien coïncider avec le déclin de ces ateliers. Tes tessons qu'on peut dater des 
années 157-158, sonl bien plus grossiers et le décor sans art. On constate ausai pour certaines productions plus courantes un retour aux formes antérieures à la conquête: la coupe campanienne à bords retournés a été imitée en vernis rouge. En 1951, la tranchée a été reprise au sud et à l'Est des emplacements déja fouillés l'année précédente. Le dallage de grès se poursuit et appartient peut-ître à la cour de l'alelier cérmique, flanqué aux angles Nord-Est et Sud-Ouest d'une construction qui a donné de très nombreux tessons. Des amorces de murailles prolongent sans doute celles précédemment dégagćes. Sous le niveau romain s'étend une couche à amphores avee un foyer assez net, des poteries et des ossements d'animaux. Doux nouveaux comptes ont été découverts ainsi que trois nouveaux noms de potiers, Auratus, Bellicuro, Tasco, el des poingons inédits: oiseau flanquant la marque de Crucuro; belluaire avec hache et bouclier; palmelte. Un tesson à engobe noir, de type italique, serait un modèle importé.

Le four de potier mis au jour à Lyon. sous le bâtiment de l'Hòtel des Postez 149 . en forme de voûte à peu près circulaire portéc sur deux murs, ćlait couvert d'une pierre plate et de trois épaisses couches superposées de terre noire rouge et jallne agglomérée avec de la paille, détail particulièrement intéressant. Un dallage de pierre appareillee marque lo niveau du sol romain et proure que le four était enterré. Cinq amphores élaient en cours de cuisson lorsque la fournée fut interrompue par la bataille de 197. T'officine travaillait pour les nrgotialores vinarii de l'île des kanabae, située sur l'emplarement actuel do la place Bellecour.

Vichy (Allier) fut aussi un centre céramique exploitant le bane d'argile plasti-

(149) P. Wullleumier, Gallia, VIII, 1950 , p. 150 . que s'étendant sur l'unc el l'autre rive de l'Allier 150. Il est caractérisé par la glagure plombifère de zes fabrications. Son activitć pourrait être antérieure à celle des officines de Saint-Rémy-en-Rollat et s'inscrire entre la fin du $\mathrm{I}^{\text {ar }}$ siècle avant l'ère el le début du II siècle de notrc ère. Lne plus grande variété de formes sc manifeste également dans les ateliers vichyssois, dont les modèles sont à rechercher dans les productions céramiques de la Haute Italie : tasses, oenochoris à panse piriforme ou pyramidale, gourdes plates, biberons, dont les décorations conservert un caractère celtique, avec leurs arcatures à pointes fleuronnées, leurs vessies de poissons, leurs chevaux à jambes humaines, les grues, les cavaliers aux boucliers ovales ou hexagonaux, les rinccaux, les godrons et les cannelures. Parmi les pièces récemment dérouvertes, on retiendra un poingen portant un ours derrière un belluaire, décochant la jambe gauche en arrière pour ćarter le fauve; des tessons représentant une fomme sur un charriot, prête à être livrée aux hê$\operatorname{tes} 151$.

I e fragment de sigillée aveo rosace de l'atelier de Censor, qui travaillait à Tròves entre 165 et 225, trouvé à Aumetz. (Moselle), est la première marque trćvire rencontrée en Iorraine. Cependant les produits de ces ateliers étaient importés à l'Ouest, en Meurthe-et-Moselle 152.

Tn catalogue des marques d'amphores d'Alise-Sainte-Reine 153 apporte des indications précises sur les relations com-

(150) Dr $\Lambda$. MoRlet, Tourisme, plages et villes d'eaux, no 30 , février 1952.

(151) Du MÈue, Vichy-Cannes, no 21, avril 1951 , p. 31-32; no 25, août 1951, p. 39-41; Plages et villes d'eaux, $\mathrm{n}^{\circ} 24$, juillet 1951, p. $37-40$.

(152) E. Delont, Gallia, VIII, 1950, p. 161163.

(I53) E. Thevenot, $X X^{\mathrm{e}}$ congr. assoc, bourguig. Semur 1949, p. 67-73. 
merciales de la cité, en rapport au $\mathrm{I}^{\mathrm{er}}$ siècle avec l'Italie qui importe ses vins en Bourgogne, au II" siècle avec l'Espagne qui lui fournit son huile. Les estampilles de Vayres (Gironde) sont assez variées, on y relève une marque de Rheinzabern 154. A la lumière des fouilles, Bavai (Nord) apparaît comme le grand marché régional de la céramique d'Argonne au re siècle 155. Ja plupart des officines, Ises Allieux, Vauquois, le l'ont des Quatre Enfants, Lavoye, Châtel, y sont représentées. Est-ce bien du vin que contenait l'amphore au nom du marchand espagnol Marcus Flavius Titurus, recueillie à Saint-Révérien (Nièvre)150 ? Très important pour l'histoire du commerce entre l'Espagne et la Gaule est le catalogue des estampilles céramiques de Tarragone $\mathbf{1 5 7}$. L'examen de ces mêmes marques pour la sigillée donne au Portugal les résultats suivants : sur 97 estampilles, 61 relèvent de J.a Graufesenque, 17 des officines de la Gaule méridionale, 5 de Montans, 3 seulement sont d'origine portugaise ${ }^{158}$. Signalons encore une étude physico-chimique de la poterie antique $\mathbf{1 5 9}$.

I.e débat sur les origines du vignoble bourguignon n'est pas clos. L'interdiction de planter en Transalpine sous la République romaine 160 avait pour objet de revaloriser les oliveraies et les vignobles appartenant aux citoyens romains. Il ne s'agissait donc que de restreindre, et non de supprimer, une culture pour pallier à

(154) A. Videau et VACHER, Rev. hist. et archéol. Libournais, 1951, p. 85-88.

(155) J. GrincourT, Gallia, VIII, 1950, p. $55-76$

(156) R. LouIs, ibid., p. 176.

(157) S. Ventura Solsona, Memorias de los museos arqueológicos provinciales, IX-X, 19481949, p. 131-135.

(158) J. M. D. OLEIRo, Revista de Guimaraes, LXI, 1951, extrt.

(159) H. Salmany, Die phisikalischen und chemischen Grundlagen der Keramik, Berlin, 1951. la concurrence indigène. Les rapports do la viticulture et de la géographie de li circulation témoignent de Ja nécessití pour les centres de production vinicole de ne point s'éloigner des routes. C'est ainsi que le vignoble autunois est un concurrent sérieux pour son voisin chalonnais: situé plus au Nord il est dans une position plus favorable a l'exportation vers les grands malrehés du vin des régions septentrionales. Ie Chalonnais: par contre dispose d'une voie navigable 161. Malgré les recherches d't. Thevenot concluant de la disparilion des annphores italiques en Bourgogne à un développement suffisant de la production locale, R. Dion $\mathbf{1 6 2}$ reste sur ses positions quant aux origines viticoles dijonnaises.

L'un des premiers résultats des fouilles sous-marines de l'épave découverte au large d'Agay-Anthéor (Var), malheureusement ridiculement pillée par les amateurs de souvenirs, a été d'apporter des documents nouveaux à l'histoire des relations commerciales de la Provence of do la Campanie au I $^{\text {er }}$ siècle avant J.-C. L'une des amphores (forme I de Iresssel), semblable à celles recueillies à Fintremont, à Fos, à Marseille et dans la cargaison du navire d'Albenga, porte l'estampille de la gens Lassia, dont l'existence est attestée à Pompéi en 2 avant l'ìre ${ }^{163}$. Parmi les objets trouvés au cours des recherches sous-marines, figure l'ancre ramence de l'île Sainte-Marguerite.. dont le jas porte une tête de Méduse en relief sur la tranche latérale, inscrite dans I!n cadre rectangulaire et dont le type s'apparente à la Vénus Rondanini (ve siècle

(160) A. AYmard, L'introduction des plantations de vignes en Gaule Transalpine sous la République romaine, dans Mél. Géograph. offerts à M. D. Faucher, I, p. 27-47.

(161) Gf. Gallia.

(162) R. Drox, Ann. Bourgogne, XXIV. 1951, p. 47-52.

(163) F. BenoIt, ibid., VIII, 1950, p. 129130. 
av. J.-G.), modèle qui persiste dans la Méditerrance hellénistique et romaine. La pièce est contemporaine de la trouvaille d'Agay 164.

C'est le "ménage" d'un campagnard fuyant devant l'invasion, à la fois vigneron et bûcheron, qui a été retrouvé dans un jardin de T'arquimpol (Mosclle) 165 , enfermé dans un chaudron de bronze recouvert par un autre plus grand et composé d'une trentaine d'ustensiles en bronze ou en fer : entonnoirs, passoires à vin dont les ouvertures dessinent des grecques, trois lampes à crochets (le copion lorrain), un plat creux cn bronzc; uac scie à archet et une scie passe-partout, deux gouges, unc tarière à douille, une enclume portative, une hache, une herminette, une hachelte à tranchant transvcrsal, une hache à fendre le bois, un ciscau, une clochelte et deux socs do charrue. Près des chaudrons avaient été déposées des chaînes utilisées pour la manipulation des troncs d'arbres et terminées tantôt par un crochet, tantôt par un anneau ovale. Isa trouvaille est à rapprocher de celle faite dans un puits à Maclaunay (Marne) ${ }^{166}$.

Le nid de statues de la rue de la République, à Orange (Vaucluse) 167 présenle. une stratigraphie assez curieuse : à un emplacement situé entrc les vestiges de murs d'un grand édificc romain, correspondant à l'interscetion des murz de la Banque Marseillaise de Crédit et de l'immeuble Nencianina, la couche de débris atteint plus d'un mètre d'ćpaisseur. Les blocs les plus importants sont à la partic

(164) Ibid., p. 130; - Rev. archéol., 1951, I, p. 223-228.

(165) E. DELoRT, Mem. acad. nat. Metz, n. s., I, 1951, p. 25; - Cahiers lorrains, 1951, p. 4148. 128.

(166) P.-M. FAVRET, C.R.A.I., 1947, p. 120-

(167) J. SAUTEL, C.R.A.I., 1951, p. 239-242; - Gallia, VIII, 1950, p. 139-141. supérieure, tandis que les morceaux de moindres dimensions, fragments de cadastres, de frises, ayant glissé entre les gros blocs, forment les assises inféricures. Puis, de part et d'autre de cette accumulation, la couche diminue lentement pour ne plus donner que des débris de placages ou des fragments peu volumineux ayant roulé sur les pentes. On peul admettre que tous ces marbres ont été déposés sur cet emplacement lors de l'abandon des monuments romains dépouillés de leurs richesses par les Barbares. Les nouveaux constructeurs d'églises avaient fait entreposer en des endroits choisis tous les débris des monuments délaiszés vraiscmblablement pour en faire de la chaux. Parmi les sculptures figurent une Vénus, réplique probable de la Vénus de Naples-Frćjus; une jambe de statue drapéc; la partie supérieure du corps d'un personnage drapé; des torses également drapés; un empercur, le torse revêtu d'une cuirasse sur laquelle un Harimaspe ćtrangle un Griffon d'une main et de l'autre poignarde un sccond Griffon, sujet peu souvent représentć mais qui se retrouve à la Villa Albani ct à Volubilis. Les morceaux de frises peuvent être rattachés à six groupes différents: Victoires ailces dirigécs vers un sujet central inconnu; personnages groupés deux à deux; nouveaux fragments de la frise de Persée, Amazones armées de lances et de boucliers s'élançant à la suite du héros; porteurs d'offrandes et animaux porteurs; frise des Centaures, tête de joueur de flûte et Cenlaure caressant une girafe. Ces éléments se rapprochent des frises de la basilique Emilia et relèvent d'un prototype commun. Ils doivent venir du théatre et représentent deux sćries chronologiqucment distinctes, attestant une réfection du monument 168. IJans les séries archi-

(168) Ch. PICARD, C.R.A.I., 1951, p. 244. 
tecturales ce sont des fûts de colonnes lisses ou cannelés, des bases, des volutes, des tailloirs de chapiteaux généralement corirthiens, de grandes architraves, des morceaux de frises unies, des corniches à modillons décorés d'oves; de rais de cœur, de denticules, des chapiteaux de pilastres corinthiens, des tables ornées de coquilles.

Contemporain du I $^{\text {er }}$ siècle avant l'ère, le cippe pyramidal de Flaux (Gard), portant la figuration, à l'intérieur d'un naislios, d'une tête sommée du calathos, témoigne une influence gréco-égyptienne ${ }^{169}$.

On retiendra parmi les récentes découvertes de sculptures et de bas-reliefs: les vestiges d'une statuette en marbre blanc, pied à brodequin à talonnettes d'une image de Mercure, à Capniac (Dordogme) ${ }^{170}$; la tête en marbre blanc d'un personnage contemporain de Néron, trouvée à Péronne ${ }^{171}$; une autre tête de même matière provenant du sous-sol d'une maison romaine de Tarquimpol (Moselle) ${ }^{172}$; le bas-relief de Saint-Cricq à Saint-Aignan (Gironde), gladiateur casqué, vêtu d'une tunique courte, les jamhes nues, marchant ì gauchr, la main droite portée en avant et tenant un bouclier ${ }^{173}$; le fragment de plinthe en marbre blanc du baptistère de Nevers, avec deux têtes juvéniles, peut-être un fragment de sarcophage allx Amours 174; on annonce ${ }^{175}$ que R. Jizop a proposé une interprétation des bas-reliefs trouvós à l'Tnstitut catholique de Toulouse.

(169) J. Jannoray, Gallia, VIII, 1950, p. 116. (170) Bull. soc. hist. et archéol. Dordogne, LXXVIII, 1951, p. 151.

(171) A. BLANCHET, B.A.C., 1943-1945, p. 335336 .

(172) R. Delort, Gallia, VIII, 1950, p. 161.

(173) B. Ducasse, Rev. hist. et archéol. Libournais, 1951, p. 6-10.

(174) R. LouIs, Gallia, VIII, 1950, p. 176.

(175) Ann. Midi, 63, 1951, p. 281.
$\Lambda$ la liste déjà longue des sculptures alexandrines découvertes dans la Gaule méridionale s'ajoute la panthère de bronze, trouvée au large de la coite méditerrancénne $\mathbf{1 7 6}$ et qui devait porter l'image d'un enfant dionysiaque se rattachant aux images de fauves rugissants. assis ou debout, la patte antírieure gauche levée, tantôt figurće isolément, tantòt associće à une figure do Dionysos. la croupe fléchissant légèrement comme. sous le poids d'un fardeau. La pièce est ì rapprocher des rondes-bosses, des petits bronzes et des monnaies, et particulièrement du Dionysos sur la panthère du Sérapéion de Memphis, contemporain du règne de Ptolémée Ier, dont le nouveau monument est très proclıe. Il faisait vraisemblablement partie de la cargaison d'un navire naufragé qu'un fragment de fanal en bronze, découpé en forme d'cnceinte de ville, crénelée et tourelée, permet de dater du rer sic̀cle de notre ère.

Trois petits bronzes figurés de Besanson (rue des Chambrettes) font partic de quelque chapelle domestique: dicu du Sommeil, avec torque d'argent, volé en 191/4 dieu assis avec orcille droite démesurée, tenant un serpent ct une grappe de raisins, type divin a rapprocher des figurines d'Amiens et de Tantilly; dieu gaulois assis dans un fauteuil d'osier, vêt.u de la pèlerine à capuchon et d'une tunique longue, à tête amovible, nouvel cxemplaire d'une série reprósentéc par les statuettes de Gotha, de l'ancienne collection Oppermann et de Nîmes $\mathbf{1 7 7}$.

Dans un puits, entre la voie romaine of la roire fut recueillic une statuctte en bronze de danseur ŕquilihriste, empreinte d'un réalisme populaire ${ }^{178 .}$

(176) R. LANTiER, C.R.A.I., 1951, p. 112-114; - Rev. des arts, 1951, no 2, p. 115-116; Mon. Piot, XLVI, 1952, p. 69-76.

(177) L. LeraT, Gallia, VIII, 1950, p. 195204.

(178) R. Louis, ibid., p. 170. 
Il n'est plus possible d'accepter lidentification proposée pour la pctite figurine de Mercure remarquée par Sauval et qui fut, en son temps, matière à poćsie. Ellc avait été trouvée dans des fouilles faites à Paris sur l'emplacement du Palais du Luxembourg, auxquelles s'intéressait $\mathrm{Ma}$ rie de Médicis. On peut se demander s'il ne s'agit pas d'un faux postérieur, exćcuté par des fondeurs italiens comme étanl plus digne d'êlre offert à la reine ${ }^{179}$.

J'Apollon citharède d'Triage (Isère), entré en 1947 dans les collections du Musce d'Art et d'Histoire de Genève ${ }^{180}$, remonte à un prototype hellénistique qui adapte l'Apollon Sauroctone de Praxitèle et le Potos de Scopas à ce nouveau sujet. La pièce, qui se date du ${ }^{\text {cr }}$ ou du $\mathrm{II}^{\circ}$ siècle de notre ère, n'est pas importée d'Italie, mais a été exécutée dans un atelier gallo-romain. Le thème représenté ctait très en faveur en Gaule, et certaine rudesse dans les traits du visage dénote un faire indigène, bien que d'une technique supérieure aux groupes des Apollons citharèdes d'Allerey, de Volesvres, de La Comelle-sous-Beuvray (Saóne-ctIoire) et de Chavériat (Jura), cuvres de bronziers chalonnais. Ce serait encore à ces mêmes ateliers qu'on pourrait attribuer les statuettes d'argent trouvées en 1764 dans la cour de l'Hôtel-Dieu de Mâcon et conservées au Musée Britannique ${ }^{181 .}$

Un fragment de la toge de la statue on calcaire de Bagnols 182, emporté, croit-on à Saugnes, a ćté retrouvé.

Est-ce bien un petit aediculum qui est, représenté sur le manche d'une patère en

(179) A. BLANCHET, B.A.C., 1943-1945, p. 176178.

(180) W. DeONNa, Museum helveticum, VIII, 1951, p. 228-234.

(181) L. Armand-Gaidiat, Rev. archéol. Est, II, 1951, p. 56-59.

(182) Bull. trim. soc. lettr., sc, et arts Lozèie, 1951 , p. 100. bronze, mis au jour à Auxerre, dans lo quartier de l'ancienne abbaye de saintJulien, au confluent du ru de Vallon et de l'Yonne près de l'écluse ${ }^{183}$ ? Vertault a fourni des appliques de serrures en tôle de bronze, des poignées de coffres faites de deux dauphins engoulant une cocquille, certainement exécutées sur place dans des ateliers dont on a retrouvé quelques moules, des creusels pour la fonte du bronze et qui ont encore fabriqué des a\})pliques zoomorphes ou anthropomorphes, tête d'animal fantastique à crêto formant anneau, masques de Faune barbu ct de silène âgó ${ }^{184}$. Rapprochées des découvertes de manches de couteaux, ces fahrications précisent lo caractère industriel de la ville gallo-romaine. Ja nature des vaissclles ef des objets en verre, perles, bracelcts, pions de jeu, fausics intailles recueillies dans les fouilles de Vertault, tendrait à prouver que les rapports commerciaux de la cité étaient plus importants avec les pays des Iellques qu'avec Alésia ${ }^{185}$.

Un mémoire posthume de F. Delage 186 traitc des arts en Limousin à l'époque gallo-romaine (Haute-Vienne, Corrèze et majcure partie de la Creuse).

In ouvrage récent $\mathbf{1 8 7}$ pose de nouveau l'épineuse question du fer à cheval. Le livre est l'œuvre d'un technicien et ses conclusions rejoignent celles que proposait, il y a vingt ans, le Ct Lefebvre des Noëttes 188 : aucun témoignage archéo178.

(183) R. LouIs, Gallia, VIII, 1950, p. 176-

(184) R. JOFFrOY, $X X^{e}$ congr. assoc. bourguig. soc. sav. Semur, 1949, p. 94-95.

(185) R. JOFFroY et R. PARIS, Bull. soc. hist. et archéol. Chatillonnais, 1950, extrt.

(186) Bull. soc. IIist. et archeol. Limousin, LXXXIII, 1951, p. 273-283.

(187) G. GARnat, Le fer a cheval a travers l'histoire et l'archéologie. Contribution a l'histoire de la civilisation, Lausanne, Spès, 1951.

(188) L'attelage et le cheval de selle d travers les ages. Paris, 1931. 
logique dûment établi, ni pour les Celtes: ni pour les Grecs, ni pour les Romains ou les Gallo-Romains, ne vient apporter la preuve de la connaissance de la ferrure à clous pour ces différents peuples. Il en est de même pour les Mérovingiens et les Carolingiens. Dans ces conditions la ferrure à clous du cheval ne peut avoir une origine antique. Elle apparaît comme la conséquence de la découverte du collier d'épaule et du développement de la chevalerie au Moyen àge $\left(\mathrm{XI}^{\circ}\right.$ siècle en Suisse). Cela ne signifie toutefois pas que les Romains ne se soient pas préoccupés de l'usure prématurée du sabot du cheval. S'ils ont ignorć la ferrure ì clous, ils ont su protéger les pieds des animaux de trait par de vóritables chaussures métalliques, les hipposandales, plaques solaires à crochets et à talonnières, fixées au sabot par des courroies, procédé encore en usage de nos jour's en Bosnie. En l'absence d'une documentation archéologique suffisante, il est encore impossible de préciser le lieu d'origine de la ferrure à clous. Un rertain nombre de raisons, dureté du sol, des routes en particulier, humidité du climat, élevage et utilisation intensive du cheval à des travaux de plus en plus lourds et rapides, principalement aux armćes, expliquent cette invention qui semblerait plutôt venir logiquement de l'Europe septentrionale que du Sud. En ménagrant les forces humaines, contraintes jusque-là d'assurer les plus lourds transportis, l'invention $\mathrm{du}$ fer à cheval a permis à l'homme d'améliorer sa condition. Fit cette dé-

couverte frappa son imagination an point d'attacher au fer à cheval des vertus magiques de protection.

7. Religion. - In essai 189 sur le caractère des principaux dieux celtiques et germaniques propose les conclusions suivantes : la plus ancienne religion de l'un ef l'autre groupe esf représentée par un culte de la fécondité. Au ciel règnenl d'une part T'eutatès, Taranis, Esus, de l'autre, Tiu, Donar et Wotan. Une divinité protectrice, la Terre Mère, est honoréc sous divers noms. Entre ciel et terre se dresse la colonne qui supporte le firmament. Aux temps próromains, il n'y a pas d'images divines, les dieux sont adorés sous la forme symbolique, roue du Soleil de 'Tiu-Teutates, hache ou doublehache de Donar-Taranis, clieval ou bûcheron de Wotan-Esus. Toutes ces figurations existent à l'âge du Bronze et peutêtre même dès le Néolithique. Au cours des siècles une óvolution se produit dans l'ordre de préséance des grands dieux: Tiu le maître du ciel cède la place à Donar, puis à Wotan, dans le panthéon germanique. Ce n'est qu'à l'époque romaine f qu'apparaît l'image cultuelle anthropomorphe empruntée à l'iconographic gréco-romaine.

Jues recherches de Fr. Benoit sur le airantire funéraire du dieu cavalier au géant anguipède et de l'écuyère Epona ont été l'objet de sévères critipues de P'. Iambrechts 190 qui se refuse à retrouver dans ces monuments toute trace de symbolisme funéraire. Ce même raractìre. pour F. Benoit ${ }^{191}$, reparaît dans un groupe de la déesse-mère et du dieu accroupi d'Autun : le dieu aux yeux clos est-il une victime propitiatoire pour le salut du défunt? la déesse-mère accueille-t-clle Is mort dans son sein ? On peut la rapprocher de la Terre-Mère de Touretles, tona!', sur ses genoux une tête coupéc. T.e dieu accroupi serait assimilé au repos

(189) F. SPRATER, Dic IIauptgötter der Kelten und Germanen, dans Festschrift Wahle, p. 206-211.

(190) P. Lambrechts, Divinités équestres celtiques ou defunts heroïsés? dans L'Antiq. class., XX, 1951 p. 107-128.

(191) F. BENoIT, Latomus, X, 1951, p. 439457. 
éternel, et la pose dans laquelle il est figuré en serait le symbole.

Est-il possible de préciser le rúle du cheval dans la religion de la Gaule orientale 192 ? On ne doit pas confondre animal et divinité. Le cheval n'est pas plus Rudiobos que Segomo et ne s'identifie pas à Epona. S'il possède un caractère sacré ce ne peut être que par un rejaillisscment sur lui-même do la nature divine du dieu dont il est le serviteur, et c'est dans celte mesure seulement qu'il serait permis d'envisager un culte du cheval. Epona n'est pas une jument divine qui a pris la forme humaine, et, hien loin d'ctre un animal, elle apparaît comme une idole féminine, une déesse à la jument. I'équidé n'est qu'un attribut comme la roue de Taranis. Son caractère n'est pas simple : à la fois génie de la prospérité et des sources, elle peut ćgalement se rattacher aux mythes d'outre-tombe. Mais il est difficilc de relier à ces croyances de l'au-delà les figurines de chevaux en terre-cuite: ce sont des cx-voto, et quant aux cavaliers en armes. le patronage de Segomo leur conviendrait mieux que celui d'Epona. Au culte des eaux se rattache le groupe du dicu cavalier au gí.unt anguipède représentant peul-être le "libérateur des eaux "193. Personnification des caux encore, mais aussi garantic de fécondité pour les humains, protectrices de l'au-delà, tels sont les caractìres des Déesses Mères 194, dont les origines remontent aux temps préhistoriques.

On ne peut nier la complexité de ces personnages divins et chacune des interprétations qu'ils provoquent contient sa

(192) E. Thevenot, Rev. archeol. Est, II, 1951, p. 129-141.

(193) Du Mene, La Nouvelle Clio, 1950, p. $603 \mathrm{sq}$.

(194) DU MENE, Rev, archéol. Est, II, 1951, p. 7-26. part de vérité. Si le dieu accroupi peut etre l'image du repos ćternel, il s'identifie aussi avec Cernunnos et on peut interpréter la pose dans laquelle il est figuré comme l'image rćalisle d'un Celte assis sur des bottes de paille, ou lui reconnaitrc un sens religieux profond $\mathbf{1 9 5}$. Un texte de Macrobe (Sal., I, 10, 21) relatif à $O p s$, interprète cctte pose comme signifiant un contact avec la Terre nourricière, et à son caractère infernal s'ajoute la personnification de la Terre, source d'abondance ct de félicité. Il en est de même du dieu cavalier au géant anguipède qui parlicipe de la nature d'Apollon-Hélios et de Jupiter-Zeus, parfois aussi figuré avec la rouc de Taranis dont il est comme l'ultime émanation $\mathbf{1 9 6}$. Cette polyvalence explique les contradictions des diverses interprétations pour deux bustes, trouvés à Mâcon en 1764, identifiés comme Apollon Grannus el Sirona ${ }^{197}$, protecteurs des chevaux au même litre que les Dioscures dans la Gaulc orientale, explication qui n'est pas généralement admise 198. Un bon exposé est donnó des cultes des Mandubiens et des Éduens 199.

Dans un pelit volume, G. Gerlach 200 retrace l'histoire des sunctuaires du Donon, plusieur's fois reconstruits of dévastés au xvinte siècle par les chercheurs de trésors et de matériaux. Il n'est pas moins difficile, au termple des sources de la Seine ${ }^{201}$ élevé entre la fin du Ier siècle

(195) Phyllis Pray Bober, American Journ. of Arch., LVI 1951, p. 13-51.

(196) J.-J. IlATT, Rev. archéol. Est, II, 1951, p. $82-87$.

(197) E. KuÜGER, Germania, 28, 1944-50, p. $213 \mathrm{sq}$.

(198) L. Arnand-Calliat, Rev. archeol. Est, II, 1951, p. 56-59.

(199) $X X^{\mathrm{e}}$ congr. assoc. bourguig. soc. sav. Semur, 1949, p. 90-92.

(200) Une montagne sacrie dans les Vosges: le Donon. Raon l'Etape, 1951.

(201) Martin, R.E.L., 29, 1951, p. 66. 
et le début du $\mathrm{Il}^{\circ}$, de reconnaître la deslination des différents édifices. Sequana apparaît comme une déesse tutélaire, protectrice du long cours du fleuve, et aussi guérisseuse. C'est aussi un temple de source qui est en cours de dégagement entre la boucle du Loing et le canal de Briare, à Montbouy près de ChâtillonColigny (Loiret), au lieu dit “Craon "202: à l'intérieur d'une enceinte rectangulaire, doublée extérieurement d'un fossé communiquant avec le Joing et renforcée de trois tours carrées sur la face orientale non défendue par la rivière, se dresse un complexe de batiments, salle octogonale abritant une piscine froide, d'autres bassins et des canalisations à l'intérieur d'une cour bordée sur trois de ses côtés d'un portique orcupé par les cellules réservées aux dévôts, qui ont laissé des ex-voto, Déesses Mères, Vénus en terre-cuite blanche. $\Lambda \mathrm{u}$ voisinage, un autre lieu de culte it triple enceinte et cella circulaire occupe le sommet d'une éminence entre la rivière et l'amphithéâtre voisin du château de Chenevières. Les fouilles du siècle dernier dans le sanctuaire du ruisseau de la Cave, à Essarois (Côte-d'Or), n'ont pas épuisé le gisement et de nouvelles recherches pourraient être fructueuses, comme le montre la découverte récente d'un nouvel ex-voto, buste d'homme assez mutilé 203. Le captage de la source de la Beugnne, au Bois Richard à Agé (Saône-etLoire) a révélé l'existence d'un nouveau temple de sources avec un petit oratoire rustique, vases, plaquette de bronze au phallus, statuettes de terre-cuite, enfant rieur, buste de femme, déesse mère assise dans un fauteuil d'osier et allai-

(202) R. LouIs, Gallia, VIII, 1950, p. 170172 ; - L. LERAT, ibid., p. 156-159.

(203) Bull. soc. hist. et archeol. Chatillonnuis, 1950-1951, p. 53. tant un enfant, monnaies des $\mathrm{l}^{\text {er }}$ et ${ }^{\circ}{ }^{\circ}$ siècles 204.

Les découvertes se poursuivent dans le sanctuaire de carriers de la montagne d'Arrée (Ariège) ${ }^{205}$ : parmi les (quatre autels de marbre de Saint-Béat dédiés it Erriapus, l'un d'eux avait été consacré par l'ensemble des marbriers travaillanl aux carrières. Il s'agit donc d'une dévolion commune à tous les marbriers, vraisemblablement groupés en collège.

Est-ce bien un temple de Mercure qui a été reconnu à l'Isle-Aumont (Yonne), près de l'église 206 ? Le culte de cette divinité a été très répandu à l'Ouest de Cihalon-sur-Saône autour d'un centre qui pourrait être fixé à Mellecey, dans la montagne et au voisinage du croisement de deur voies romaines ${ }^{207}$. Ce n'est pas une villa qui a été découverte à Thiverny (Oise), mais peut-être un sanctuaire, avec peinturess, rattaché à un très ancien lieu de culte ${ }^{208}$.

La mise au jour ì Nîmes, boulevard Gambetta 209, d'une mosaïque à six médaillons dans un encadrement de grecques et cantonnés aux angles de fruits, de poissons ou d'oiseaux encadrant un motif central, Bellérophon et la Chimère, sujet rarement traité par les mosaïstes, apporte des précisions sur le caractère eschatologique de ces pavements au $\mathrm{III}^{\circ}$ siècle de notre ère. Autour du mythe de Bellérophon, utilisé dans le décor des sarcophages (Port-Gueydon), sont associés des motifs prophylactiques, personnages empruntés à la marche du temps, Vents à Avenches, Saisons à Nîmes, paon

(204) L. Armand-Caldiat, Rev, archeol. Est, II, 1951, p. 60-61.

(205) M. Labrousse, Rev. de Comminges, XLIV, 1951, p. 74-82.

(206) J. SCAPUla, Gallia, VIII, 1950, p. 81.

(207) L. Armand-Calliat, B.A.C., 1943-1945, p. 167-169.

(208) Comm. des fouilles, 27 mai 1952.

(209) R. LANTiER, C.R.A.I., 1951, p. 48-52. 
enblème de la résurrection, cratère du breuvage d'immortalité, canards symboles de l'hiver, décors centrés sur l'idée de la mort et de l'âme promise à l'ćternité des joics célestes. Cette tendance de l'art romain, aussi éloignée que possible d'un esthétisme gratuit, se manifeste encore dans les représentations de scènes cmpruntées à la chasse 210 . Ce qui importe dans la poursuite du lièvre sur le sarcoplage de Jovin (E. 367), chasse d'origine gauloise, c'est bien moins la conformité de la scène à la réalité ou sa beauté propre, que la signification qu'elle comportc et le bénétice surnaturel qui peut en résulter, tant pour le spectateur que pour l'auteur du monument. Cette même eschatologie reparait sur la statue de sphinge assise les jambes repliées d'AvalIon, sur la pierre d'Estrains (Nièvre) et le groupe roman de Flavigny (Côte d'Or), louve tenant un animal dan̈ ses grriffes 211 .

Au bois d'Yc̀vres, dans la boucle du Cher et de l'Yèvre, avant le confluent, on mit au jour une statuetle d'homme debout, brisćc aux genoux. tenant des fruits et des pommes de pin dans un pan de sa chlamyde, peut-être Sabazius, témoignant ainsi d'une certaine diffusion de la religion anatolienne chez les Bituriges 212. Divers autels dédićs à Mercure et à un Génie ont été recueillis à Villars 213 ct à Cururon (Vaucluse) 214 .

Une interprétation nouvelle est donnée des sujets figurós sur l'œnoché en verre bleu, découverte jadis à Resançon au

(210) J. Aymard, Essai sur les chasses romaines des origines a la fin du siecle des Antonins, Bibl. Ec. fr. Athènes et Rome, fasc. CLXXI, 1951; - cf. G. Gh. PICARD, Journ. Sav., 1951, p. 72-85.

(211) M. RENARD, XXe congr. assoc. bourg. soc. sav. Semur, 1949, p. 93.

(212) R. LouIs, Gallia, VIII, 1951, p. 175,

(213) J. SAUTEL, ibid., p. 138.

(214) Ibid., p. 138. quartier de Ghamars; scène de purification au cours d'une initiation aux mysteres de Dionysus ${ }^{215}$.

Il est difficile de donner une explication du sujet représenté sur un relief de marbre blanc, longue tige et a gauche les restes d'un avant-bras, à droite les deux mains d'un autre personnage tenant une coupe à anses, trouvé à Chartres, rue du Cardinal-Pie 216. Culte des arbres ?

Sur la colline du Castellar, ì Istres (Bouches-du-Rhône), une dédicace aux Matrones a été relevée 217.

Dans le folklore, les mythes galloromains ont laissé des survivances plus ou moins estompées, en particulier dans le comporterncnt des jeunes gens à l'ćgard des jeunes filles, et le 1er Mai évoque l'histoire galloise de l'héroïne Riannon et. peut-être d'Epona ${ }^{218}$.

8. Numismatique. - Si l'on examine l'ensemble des monnaies trouvées à Saint-Bertrand-de - Comminges (Haute Garonne) depuis un quart de siècle, on constate tout d'abord les apports des ateliers de Tarraconaise, de la vallée du Rhóne et de la Cyrénaïque. Sous le HautEmpire, co sont des pièces do Rome avec le sigle S.C. Quant au numéraire du BasEmpire, il est originaire des officines de Trèves, d'Arles et de Lyon 219.

Des trésors monétaires ont été trouv'és à Montbouy (Toiret) autour de la ferme. de Craon, vase en terre-cuite ayant contenu 5.8.89 monnaies de Gordien III à Trébonien Galle et aux deux Tétricus, les émissions locales y sont nombreuses 220 ;

(215) M. DAYET, Rev. archeol. Est, II, 1951, p. $40-43$.

(216) A. Blanceet, B. A. C., 1943-1945, p. 189-190.

(217) F. Benort, Gallia, VIII, 1950, p. 123.

(218) $\Lambda$. GRENIER, Rev. hist., GGIV, 1950. p. 28-29.

(219) P. SAPÈNE, Rev. Comminges, XLIV, 1951, p. 139-154.

(220) R. LoUIS, Gallia, VIII, 1950, p. 171. 
en Normandie ce sont les découvertes d'un grand bronze de Philippe Ier à Falaise 221, du Manoir, à Rycs, cachette de 425 pièces de Vespasien à Gallien, comme à Estréesla-Campagne, enfermées dans un vase de terre-cuite 222. Ia cachettc de la rue Allix a Senlis était constituée de 1168 petits bronzes. principalement des antoniniani de Valérien, de Gallien, de Saloniri, de Valérien II, de Claude II, de Quintille, d'Aurélien, de Postume, de Marins, de Victorien ol de 'Létricus, frappées à Rome, à Milan et dans les ateliers locaux de Cologne et de Trèves. Le trésor avait été enfoui sous le règne d'Aurílien, aux appruches de la grande invasion de 275, an bord d'une route, dans une hourse fermée de cordons. I a composition de la cachette rappelle celle du trésor de Ia Vineuse, dissimulé au plus tôt sous Probus et comportant des monnaies gardées en attendant des temps plus favorables à leur négociation, ou parce que la circulation du numéraire antérieur à la réforme d'Aurélien avait été prohibée 2:4. T.e pot de terre trouvé à 80 mòtres à l'Ouest de l'úglise d'Hamel-Bouzancourt (Somme). dans une villa gallo-romaine 225 , abritait 216 pièces, dont 1 monnaic gauloise et 215 deniers d'argent de Faustine la Jeune à Gordien III, représentant les ćcnnomies d'une famille de paysans qui l'avaient enfoui lors de la disparition de l'or pour conserver des monnaies de bon aloi 226 . Parmi les monnaies antiques recueillies au Mont-Héraple (Moselle), figurent un médaillon de Périnthe, en Thrace, de

(221) Dr. Donanlo, Bull. soc. antiq. Normandie, LI; 1948-1951, p. 268.

(222) Ibid., p. 350-351.

(223) R. Louis, Gallia, VIII, 1950, p. 179

(224) J. Lafaurie, Bull. soc. fr. numism.,

5, 1951, p. 2-3.

(225) J. Heurgon, Bull. trim. soc. antiq. Pirardie, 1951, p. 14-21.

(226) lbid., p. 67. l'époque de Septime-Sévère et un módaillon de Constant I, en argent, frappé à siscia (Pannonie) 227.

\section{VI}

TEMPS CHRÉTIENS HT MÉlROVINGIENS

Jeux épitaphes du cimetière de SaintLaurent à Iyyon ${ }^{1}$, datées des années 652 653, e'est-ì-dire de la $17^{\text {e }}$ annćc du l'ègne de Clovis II, fournissent l'une des rares dates auxquelles on peut sûrement accrocher la chronologie mérovingienne du viI siècle, découverte lourde de conséquences permettant de fixer vers 531 la date du meurtre des enfants de Clodomir et de reporter le mariage de clovis et de Clotilde antérieurement à l'annće 500 c'està-dire à la guerre contre les Burgondes.

I.e regretté $\mathrm{F}$. Lot a donné une seconde ridition de son ouvrage, La fin du monde antique et lo début du Moyj'n âge 2.

L'histoire des origines de l'égrlise de France préoccupe de nombrcux auteurs. Jusqu'au milieu du Iv $^{\mathrm{e}}$ siocle les documents relatifs aux premiers temps des ćglises épiscopales restent rares et les discussions sur les légendes do l'apostolicité de ces ŕglises sont dísormais inutiles. $\Lambda$ côté de pièces d'une authenticití certaine (lettres des chrétiens de Iyon et de Vienne, de Saint-Gyprien au Pape k.tienne), bien d'autres ne contiennent que des données imprécises, tels les catalogues épiscopaux. Quant aux renseignements apportés par l'archéologie, leur inlerprótation reste toujours délicate et la topographie est à utiliser avec prudence.

(227) J. BABELON, B.A.C., 1943-1945, p. 252253.

(1) C. Countors, Mel. L. Ilalphen, p. 155164.

(2) L'évolution de l'Ilumanité. Paris, 1951. 
Par contre, on dispose de sources plus abondantes et variées, témoignant de la vitalité de la Gaule des invasions ${ }^{3}$, qu'étudie D. Rops, sous le titre : L'église des temps barbares 4.

Dans notre Midi, ce n'est qu'à partir de 445 que les ćvèques d'Aix échappent à la primatie d'Arles et commencent à jouir sans contestation de la juridiction métropolitaine sur la Narbonnaise seconde, constituée sous le principat de Gratien. Il ne semble pas, comme le supposait Mgr. Iuchesne, que la nouvelle province ait été affectée par la décision prise, en 450 , par lc Pape Lcion de reconstituer une province ecclésiastique arlésienne. On cn aurait pour preuve le conflit qui, entre 450 et 461, amène le siège d'Embrun à lutter à son tour pour établir sa propre juridiction sur les évêchés des Alpes-Maritimes 5. L'évêque Crescent nommé dans l'Épître de Saint Paul aux Galates, épître écrite en róalité pour la Gaule méridionale, siégeait à Vienne sous le règne de Néron 6, renseignement important pour l'histoire de l'organisation ecclésiastique du pays au rer sic̀cle de notre ère. I.a vie chrétienne en Bourbonnais 7 au vi siècle, d'après les renseignements donnés par les Vies de Saint I,upicien, do Saint Pourcin et de Saint Patrocle, se serait ordonnce autour de trois centres principaux. J'introduction et la propagation du cultc de Saint Pierre ${ }^{8}$ dans le diocèse de Metz remonte aux premiers temps chrétiens et à l'époque franque pour atteindre son apogće sous les Carolingiens. Après Saint

(3) E. Griffe, Rev. de l'eglise de Fr., XXXVII, 1951, p. 40-52.

(4) Paris, A. Fayard, s. d. (1950).

(5) E. GRIrre, Bull. de littér. ecclésiastique, LI, 1950, p. 65-74.

(6) Chanoine BouIsson, Rhodania, 1948, p. $27-29$.

(7) F. Laroque, Bull. soc. d'hist. et d'archéol. Vichy..., 1950, p. 259-263.

(8) Chanoine Morhain, Mem. acad. nat. .1/ctz, n. s., 1951, p. 12.
Pierre et la Vierge, Saint Martin 9 est, dans la Moselle, le plus fréquent des patrons des églises. Protecteur de la dynastie mérovingienne, il était venu à Trèves en 371 et 384 , passant par Toul et sans doute par Metz. Son culte est localisé le long des rivières, plus rarement sur les routes romaines et dans les régions forestières, favorisé par les rois d'Austrasie et les scigneurs francs. Dans la Gaule septentrionale 10 , le ròle des monastères de Saint-Waast, de Saint-Amand et de Saint-Bertin a été prépondérant dans la diffusion de l'art et de la civilisation, avant comme après les invasions normandes.

L'importance de cette action du monachisme a été bien misc en valeur à l'occasion du XIV centenaire de la naissance de Saint Colomban : rôle des abbayes dans la formation des cités ${ }^{11}$; rapports du saint et de la Gaule franque ${ }^{12}$; les moines irlandais en Gaule avant Saint Colomban; démêlés du saint avec l'épiscopat franc; influence de Colomban et de ses compagnons sur les monastères de la Brie, Farmoutiers, Jouarre, Saint-Faron de Meaux, Chelles 13; fondations colombaniennes dans le diocèse de Bourges 14; Saint Golomban et l'Alšace ${ }^{15}$, son voyage en remontant le Rhin de Mayence en Suisse, séjour à Strashourg. I'action colombanienne semble s'être exercée principalement par ses disciples, d'ailleurs assez faiblement, mais son culte aurait été assez en honneur en Alsace 16. Cette influence du monachisme anglo-saxon en

(9) Du MÈME, ibid., p. 16-17.

(10) J. LeSTOCQuoy, Bull. soc. d'ét. prov. Cambrai, XLIII, 1950, p. 1-11.

(11) Du MÊme, Mél. colombaniens. Actes du congrès internat. Luxeuil, 1950.

(12) R. LAPRAT, ibid., p. 119-141.

(13) A. Barrault, ibid., p. 197-208.

(14) J. MELLOT, ibid., p. 209-210.

(15) R. METZ, ibid., p. 217-232.

(16) M. BARTH, ibid., p. 259-275. 
Occident se manifeste avec force au cours des derniers temps mérovingiens, pendant lesquels l'épiscopat ne joue pas un rôle prépondérant. Plus missionnaire que contemplatif, il se mêle au monde pour y exercer son action. La réforme de Benoit d'Aniane, tendant à séparer le moine du monde et à le vouer surtout à la prière liturgique, eut pour conséquence la disparition de son influence en faveur du pouvoir épiscopal 17, fort important all début de l'époque mérovingienne, alors que l'évêque apparaît comme le defensor civitatis.

Des fragments historiques de Paulin de Nole conservés par Grégoire de Tours retracent en effet les efforts faits, en 407409, par les évêques du Sud-Ouest de la Gaule pour venir en aide à leurs ouailles victimes de l'invasion des Vandales 18. Un évêque, Saint Arnould de Metz, et ses successeurs ont joué un grand rôle à épinal dans la fondation du bourg, du chapitre de Saint-Goëry et de la hasilique Saint-Maurice 19.

On lira volontiers le mímoire que le chanoine Coolen 20 consacre ì l'examen de l'attitude des chrétiens de Gaule au vir' siècle, se tenant mal aux offices, encore empreints d'un paganisme latent, au point d'obliger le clergé à christianiser les anciennes fêtes païennes, ignorants et de mcurs rudes. T'église est alors, autant un licu de réunion oì l'on traite ses affaires, qu'un sanctuaire.

Il sera nécessaire de soumettro à de nouvelles enquêtes le problème de la rógression urbaine pendant le Bas-Fmpire. Colle-ci ne parait pas preisenter le carac192

(17) Et. Delaruelde, Mél. L. Halpen, p. 185-

(18) P. Courcelle, ibid., p. 145-153.

(19) R. Pestart, Ce qu'il faut savoir des origines et du passé de la ville d'Épinal, Nancy, 1951.

(20) Bull. trim. soc. acad. antig. Morinie, XVII, 1951, p. 449-470. tère catastrophique qu'on lui prâte et l'exemple de Iutèce ${ }^{21}$ serait en faveur de cette révision. Vers 280 , les beaux quartiers ont été ravagés par l'incendie, mais au Nord à Saint-Gervais, un faubourg assez misćrable se constitue autour d'un martyrium. Dans l'île, c'est la ville close; au Sud, sur les pentes septentrionales et méridionales de la Montagne Sainte-Geneviève, entre la Seine, les arènes et la Bièvre, la ville ouverte, le virus, se temminait par un vaste cimetière, lieu de pèlerinagc attirant les fidèles sur la tombe de SaintMarcel. Le IV siècle marque le développement des faubourgs. Les pertes sont alors comblées et la vie s'organise de nouveau autour des paroisses suburbaines. La prépondérance de la rive gauche s'affirme aux $\mathrm{VI}^{\mathrm{e}}$ et vire siècles, et la rive droite participant à cet essor, c'est une véritable ville que les Mérovingiens lègueront aux Carolingiens. Le tableau n'est pas très différent si l'on envisage le problème du peuplement de la civitas des Parisii, oì trois régions avaient particulièrement relenu l'attention des propriétaires terriens gallo-romains : les bords de la rive gauche de la Seine, la vallée de la Marne entre Saint-Denis et Sevran, le cours moyen de la Marne. En somme, peu de changements ont été apportés par la conquête franque dans l'occupation du sol et sa mise en valeur, si ce n'est une transformation de l'état de la propriété. Ton élément nouveau, l'orientation du trafic vers la vallée du Rhin et la Germanic, va amorcer le développement de l'agglomíration de la rive droite qui tend alors à se poser en rivale de l'autre hourg.

Poitiers 22 a cessé d'être une grande.

(21) M. Roblin, R.E.A., LIII, 1951, p. 301311; - Du Même, Le terroir de Paris aux époques gallo-romaine et franque. Peuplement et defrichement dans la civitas des $\mathrm{Pa}$ risii. Thèse, Paris, 1951.

(22) M. GÉraud, Mél. L. Halphen, p. 271279. 
ville depuis la seconde moitić du III siècle. Ses monuments ont servi de carrière pour la construction de l'enceinte sur la partie orientale du plateau, cntre les vallées de la Boivre et du Clain, entourée par les ruines des thcrmes sur la partie orientale du plateau, des restes des arènes et des quatre cimetic̀res, camp r'elranché flanqué dans la banlieue d'ouvrages défensifs et rósidence du conmandant des Tarifates et des Sarmates en Poitou. Le christianisme y marque sa forte empreinte el la vie s'organise. la aussi, dans les bas quartiers autour du baptistère Saint-Jean. Déjà la cathédrale occupe son emplacement actuel et un monastère sera foudé par sainte-Radegonde, tandis que les basiliques de SaintHilaire de la Cellc, de Saint-Savin deviendront des centres de pèlerinages. I)e ces deux exemples il est difficile de conclure de l'étroitesse du castrum à la faiblesse du peuplement. La ville close ne représente que la nócessité de posséder un refuge fortifié rapidement établi.

Jusqu'au ve siècle, la vie se poursuivra à Arles dans son cadre ancien ${ }^{23}$. Au quartier de l'Auture, entre l'angle Sud-Est du castrum et l'amphitéâtre, Saint Césaire construit, au IV siècle, une abbaye de religieuses. $\Lambda$ u siècle suivant, l'épiscopium est transféré au forum. Un quartier chrétien se dessine, semblable à ceux de Djemila, de Timgad et de Salone, avec ses chapelles, ses oratoires, son baptislère, ses églises succédant ì des lamples: païens. Le repliement est caractéristique. I'ecclesia qui n'est pas celle du couvent, mais la seule église de la cité au Iv siècle, constitue dans l'abbaye une des parties d'une basilique triple vors la seconde moitié du ve siècle, église abbatiale et, bien qu'interdite aux gens du monde, on s'y fait enterrer ad sanctos. D'abord pla-

(23) F. Benort, Cahiers archéol., V, 1951, p. 31-59. cée sous le vocable de Notre-Dame, la cathédrale sera dédiée à Saint titienne, et le nom de Saint Trophime associé à celui du protomartyr préciser'a le penchant du clergé à substituer une légende folklorique à l'hagiographie liturgique. Sa construction ramène aux temps de la splendeur de la cité sous le règne de Constance et le pontificat de \%ozime. L'abside d'une église paléochrétienne 24 a été dégag'ée à l'angle sud-Est de la cité, empâtée dans un chevet à trois pans, décorée intérieurement d'une colonnade et de placages de marbres du IV siècle, au voisinage du vieux baptistère édifié à la périphérie, restes de la cathédrale du ${ } V^{e}$ siècle ou de l'église abbatiale consacrée par Saint Césaire en 514.

Une nouvelle précision est apportée à la topographie chrétienne d'Auxerre 25. Saint Germain n'ayant pas résidé à Térins, on peul recomnaîlre dans l'insula Aralanensis le site du monastère fondé par le saint aux portes de la cité, peut-être à Orgelaine, un lieu-dit de la rive droite de l'Yonnc.

Des constructions de caractère religieux ont été découvertes à Dixmonts (Côtc d'Or) ${ }^{26}$, à Lescar (Basses-Pyrénées) ${ }^{27}$, et sous l'église des Templiers à Saint-Raphaël (Var) 28 une crypte próromane. $\Lambda u$ sujet de ces types de construction, J. Hubert 29 remarque que durant l'époque mérovingienne les corps saints les plus vénérés, ceux de Saint Martin, de Saint Denis, de Sainl Germain d'Auxerre, de Saint Julien de Brioude, n'avaient pas été déposés dans une crypte, mais sur le sol même de la basilique. Le but recherché par l'édification de cryptes plus vastes que

(24) Du MÊme, Gallia, VIII, 1950, p. 120.

(25) R. Louis, Mél. L. Halphen, p. 445-451.

(26) Ann. Bourgogne, XXIV, 1951, p. 82.

(27) B. G. ANDrat, Bull. soc. lettr., sc. et arts Pau, XI, 1951, p. 27-31.

(28) F. Benort, Gallia, VIII, 1950, p. 128.

(29) J. Hubert, Mél. L. Ilalphen, p. 351-357. 
les confessions des églises romanes, mais aux entrées plus étroites, pour y placer le tombeau du saint, est de rendre celuici plus diflicilement accessible aux foules. D'autre part, le partage des reliques cntre crypte haute et íglise basse permet de donner satisfaction à toutes les dévotions.

Le baptistère de Saint Jean à Poitiers 30 a été plusieurs fois remanié : au IV siècle il faisail partie du groupe épiscopal. Reconstruit en son état actuel au vir" siècle à usage de baptistère, il est alors éloigné de la cathédrale, car on a cherché à le reconstruire sur son emplacement primitif. Très différent des baptistieres provençaux, il se rapproche du temple païen, mieux encore d'un tombeau antique, tel celui de Galla Placidia à Ravenne. A l'intérieur, la piscine octogonale est de forme classique. L'emploi du marbre de SaintBéat, dans le décor des absidioles au vir siècle, témoigne de la réputation des aleliers pyrénéens. Des sondages au baptistère de la cathédrale de Nevers ont fait connaître la présence d'un drainage du $\mathrm{VI}^{\circ}$ siècle, destinć à combaltre l'humidité du sous-sol 31, excluant l'existence d'une cuve baptismale au-dessous de re niveau. celle-ci ćlail incluse dans un socle polygonal où elle a été retrouvée, do forme eirculaire, légèrement ivasée de has en laut, le fond recouvert d'une dalle plate I.e socle appartient à une restauration du r.e sic̀cle. Les portes à encadrements de dalles plates sont contemporaines de l'édifice primitif.

Ce sont les découvertes et les fouilles dans les cimetières mérovingiens qui sont oncore les plus nombreuses cette année: sarcophages paléochrétieıs atux Aliscamps autour de Saint Honorat 3:; - sépulture.

(30) Ann. Midi, 63, 1951, p. 122-125.

(31) F. Benort, Gallia, VIII, 1950, p. 119.

(32) Dr. Segui, Bull. soc. d'ét. scientif. Aude, LI, 1951, p. XXIV. à Treixan (Aude), entourée d'une murette de galets roulés, avec tessons grossiers, cendres ct charbons de bois 33 ; - deux sarcophages brisés en pierre de ciouze, près du prebytère d'Aymet (Dordogne) 34; - sarcophage sur la route de Châteaul'Ermitage à Saint-Biez-en-Belin (Sarthe) $\mathbf{3 5}$; - nouveaux sarcophages à Vernon (Eure), rue Carnot dans l'axe de la rue Saint-Jacques ${ }^{36}$, et cinq inhumations avec ou sans cercueils de bois, appartenant au vaste cimetière qui s'étend jusqu'à la place de Paris, antérieur à la construction de la primitive iglise et $d u$ remparl; - sarcophages en place à NotreI)ame-de-l'Isle (Eure) dans le jardin de la cure et inhumation en pleine terre ou dans des cercueils de bois ${ }^{37}$; - squeletle sans mobilier à Louviers (Eure), rue du Mûrier, dans un sarcophage en pierre de Saint-T,eu, où d'autres sépultures avaient élé trouvées antérieurement 38 ; - tombes isolées dans le cimetière franc de Tournedos-sur-Seine (Eure), avec entourage de plâtre et mobiliers, plaques-boucles en fer, scramasaxe, couteau en fer ${ }^{39}$; - cimetière à Champigny-sur-Yome et à Dollot, commune de Chiroy (Yonne) ${ }^{40}$; plus de 350 tombes ont été fouillées sur la butte de l'Isle- $\Lambda$ umont ( $\Lambda$ ube) 41 : les plus anciennes du $v^{r}$ siècle au Nord, puis šctendant jusqu'à l'église, sont disposées par rangées, tantìt en pleine terre, tanlit dans un sarcophage en matériaux de remploj ou dans un cercueil de bois. Du $\mathrm{VI}^{\mathrm{e}}$ au vill ${ }^{\mathrm{e}}$ siècle le mode funérairc le plus fréquent est le sarcophage en pierre

(33) E. Vautier, Bull. soc. hist. et archéol. Périgord, LXXVIII, 1951, p. 117.

(35) La Province du Maine, 2 sér., XXXI, 1951, p. 203.

(36) A.-G. Poulati, Bull. soc. normande ét. méhist., XXV., 1950, p. 50-51.

(37) Ibid., p. 52-53.

(38) M. Тнiвout, ibid., p. 58.

(39) A. DIVRY, ibid., p. 58

(40) Ann. Bourgogne, XXIII, 1951, p. 68.

(41) J. SCAPUla, Gallia, VIII, 1950, p. 81-84. 
de Châtillon-sur-Seine à décor de stries horizontales ou de croix pattées. Les mobiliers ne sont que le complément de. l'mhumation habillée et les bouc.les damasquinces apparaissent avec le VII ${ }^{\mathrm{e}}$ siècle. La multiplicité des inhumalions à l'intérieur d'un même sarcophage témoigne de la prépondérance de l'élément gallo-romain dans la population. Unc zone dépourvue de sépultures. avec puits à eau, correspond à l'emplacement du monastère. $\Lambda$ près sa destruction au $\mathrm{Ix}^{\mathrm{e}}$ sic̀cle par les Normands, on contiiule a inhumer sur la butte sans ordre et en superposition. Les sarcophages en pierre sont remplacés par des cercueils de bois et les mobiliers sont rares. Sous les premiers Carolingiens, la bulle redevient unc place-forte; - 45 tombes à gauche de la route de Gyé-sur-Seine, à Loches-surOurce (Aubc), contenant des mobiliers des $\mathrm{VI}^{\mathrm{C}}-\mathrm{VHH}^{\mathrm{e}}$ siècles ${ }^{42}$, à l'intéricur de vastes fosses, tantôt avec cercueils, tantôt avec recouvrement du corps par des mottes do terre gazonnées, traces de feux rifuels; - cimetière à Hoërecourt (HauteMarne), dans la plaine de la Marne, avec sarcophages et stèles à sommet arrondi, acrotères et croix de Saint-Andrć, poteries à décors à la roulctte 43 ; - tombe près de la chapelle de Saint-Martin à Taulignan (Drôme) ${ }^{44}$ et à Crussols ${ }^{45}$; 44 nouvclles sépultures à Curtil-sousBurnand (Saône-et-Ioire) contenant des plaques richement ornées $\mathbf{4 6}$; - sarcophages avec poisson dans l'église de cheminot (Moselle) 47 .

(42) DI MÈME, Rev. archéol. Est, II, 1951, p. 143-151.

(43) V. StChepinskr, Cahiers Haut-Marnais, 1951, p. 103-107.

(44) Bull. soc. d'archéol. et de statisq. Dróme, LXXI, 1951, p. 249.

(45) J. SAUTEL, Gallia, VIII, 1950, p. 134

(46) M. LAFOND, Rev. archeol. Est., II, 1951, p. $62-63$; - P. WuIlleumier, Gallia, VIII, 1950 , p. 153.

(47) Les cahiers lorroins, 1951, p. 22.
On commence à étudier les centres de fabrication des sarcophages non décorés, dont les emplacements sont précisés par la toponymie moderne ${ }^{48}$, Les Serqueux, Cercoux, Cercueil, Sercour, Sarcus, Sercus. A Quarré-les-Tombes (Yonne), la centaine de cuves vides trouvéc autour de l'église roprésente le stock d'un immense dépôt. Les entropreneurs prenaicnt à leur charge l'extraction des blocs de calcaire, dont une carric̀re utilisée dans ce but a été reconnue à proximité des groltes d'Arcy-sur-Cure (Yonne) près de la fontaine de Saint-Moré 49.

Ians les sites funéraires, on constate, ol ccla dès la fin du Iv' siècle, un amenuisement du mobilier funéraire qui n'est plus qu'un accessoire de l'inhumation habillée, ou l'expression d'un rite païen en voie de disparition sous l'action du christianisme 50. Quant à l'inhumation on terre bénie par l'église, dans les cimetières s'ćtendant autour des édifices du culte, elle repose sur la conviction que le corps du trépassé est sanctifić par la grâce divine et, comme le croyaient aussi les zélateurs de Mithra, destiné à resssusciter, cc qui exclut toute idée de souillure. C'est pourquoi on inhumait sans scrupules mêtme à l'intérieur des églises. De même que d'autres rites chrétiens, celui-ci a ses antécédents dans les cultes mystiques chrétiens. Le dépôt des cendres dans un téménos consacré à une divinité secourable a précédé l'inhumation en terre bénie par l'église ${ }^{51}$. La présence de feux rituels allumés sur ou au voisinage de la tombe, est encore une survivance du paganisme qui n'est pas sans rapport avec le rite de

(48) P. LEBEL, Rev. archéol. Est, II, 1951, p. $166-171$.

(49) A. Leroi-Gourhan, Gallia, VII, 2, 1949, p. 247.

(50) E. SALIN, C.R.A.I., 1951, p. 123-128.

(51) W. Volgraf, Mém. présentés par divers sav. a Acad. Insc. et B.-L., XIV, $2^{\mathrm{e}}$ part., 1951 , p. $395-396$. 
l'incinération (inhumation partielle et feux rituels au Totenberg, à Heidenheinı, Wurtemberg). Bien que rare, le rite de l'incinération n'a pas été inconnu du monde mérovingien, où il apparaît en GrandeBretagne, plus exceptionnellement en Gaule septentrionale 5:.

Une contribution importante à l'étude de la symbolique du Haut Moyen àge est domnéc par un mémoire pour le diplòme d'éludes supérieures d'histoire, présenti levant la Faculté des Iettres de Toulouse par Jean Boube, I. chrisme en Gaule et son évolution, inventaire pratiquement complet de tous les chrismes antérieurs au XII siècle, avec essai de datation, classement morphologique et géographique 53 .

Une fibule a été trouvée dans le cimctière mórovingien de St-Aubin-sur-Halgot (Calvados) ${ }^{54}$, et une description exacte a été donnée de boucles en fer damasquini de Boussières (Doubs) : Daniel entre les lions, earactérisé comme sur la plaquehoucle du Gras Châlet (Suisse) par les proportions démesurées de la tête du híros 55.

On a beaucoup discuté sur le mode d'utilisation de la francisque, à la fois

(52) E. SALiN, Mél. L. IIalphen, p. 637-640.

(53) J. Boube, Rev. hist., CCVI, 1951, p. 181.

(54) F. Rault, Bull. soc. antiq. Normandie, LI, 1948-1951, p. 406.

(55) E. SALIN, Rev. hist. Lorraine, 87, 1950 , arme de guerre et outil de campagne, dont le tranchant fait avec l'axe du manche un angle pouvant atteindre $125^{\circ}$. Partant de ('es donnćes, $A$. Thouvenin 50 reconstitue un exemplaire de la francisque et retrace par des graphiques les gestes nécessités par le maniement de la hache de jet.

Trouvé près de l'épaule droite du squelette, dans une sépulture en lerre libre, le casque de Tróvières (Hainaul) se ratlache a la série qui a pour prototype le casque de Baldenheim. Mais l'exemplaire bolge ne porte aucun décor et sa calotte mćtallique devait être recouverte de cuir. C'est unc arme de combat, forgée par un artisan pour un guerrier déterminé, et non un casque de parade. Il n'est pas alors nécessaire de rechercher, pour cet objet, une origine orientale. On connait, en effet de nombreux textes mentionnant le cassis. casque de fer, et la galea, casque de cuir. Ce dernier modèle est connu dòs le BasFmpire (casque de cuir de Monceau-leNeuf) et le casque de Trévières se rattache à ces fabrications ${ }^{\mathbf{5 7}}$.

\section{Musée des Antiquités Nalionalıs:} Décembre 1952.

Raymond IANTIER.

(56) A. Thouvente, ibid., p. 23-28.

(57) G. Faider et $\Lambda$. France-Lanord, Rer. belge d'archéol. et d'hist. art, XX, 1951, p. 265-272. 